\title{
Very Large Telescope three micron spectra of dust-enshrouded red giants in the Large Magellanic Cloud
}

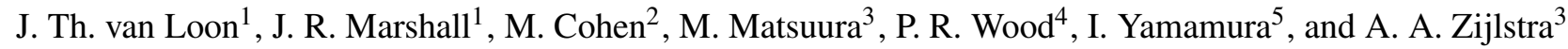 \\ 1 Astrophysics Group, School of Physical \& Geographical Sciences, Keele University, Staffordshire ST5 5BG, UK \\ e-mail: jacco@astro.keele.ac.uk \\ 2 Radio Astronomy Lab, 601 Campbell Hall, University of California at Berkeley, Berkeley CA 94720-3411, USA \\ 3 Department of Physics and Astronomy, University of Manchester, Sackville Street, PO Box 88, Manchester M60 1QD, UK \\ 4 Research School of Astronomy and Astrophysics, Australian National University, Cotter Road, Weston Creek, ACT 2611, Australia \\ 5 Institute of Space and Astronautical Science, 3-1-1 Yoshinodai, Sagamihara, Kanagawa 229-8510, Japan
}

Received 18 September 2005 / Accepted 17 October 2005

\begin{abstract}
We present ESO/VLT spectra in the 2.9-4.1 $\mu \mathrm{m}$ range for a large sample of infrared stars in the Large Magellanic Cloud (LMC), selected on the basis of MSX and 2MASS colours to be extremely dust-enshrouded AGB star candidates. Out of 30 targets, 28 are positively identified as carbon stars, significantly adding to the known population of optically invisible carbon stars in the LMC. We also present spectra for six IR-bright stars in or near three clusters in the LMC, identifying four of them as carbon stars and two as oxygen-rich supergiants. We analyse the molecular bands of $\mathrm{C}_{2} \mathrm{H}_{2}$ at 3.1 and $3.8 \mu \mathrm{m}, \mathrm{HCN}$ at $3.57 \mu \mathrm{m}$, and sharp absorption features in the 3.70-3.78 $\mu \mathrm{m}$ region that we attribute to $\mathrm{C}_{2} \mathrm{H}_{2}$. There is evidence for a generally high abundance of $\mathrm{C}_{2} \mathrm{H}_{2}$ in LMC carbon stars, suggestive of high carbon-to-oxygen abundance ratios at the low metallicity in the LMC. The low initial metallicity is also likely to have resulted in less abundant HCN and CS. The sample of IR carbon stars exhibits a range in $\mathrm{C}_{2} \mathrm{H}_{2}: \mathrm{HCN}$ abundance ratio. We do not find strong correlations between the properties of the molecular atmosphere and circumstellar dust envelope, but the observed differences in the strengths and shapes of the absorption bands can be explained by differences in excitation temperature. High mass-loss rates and strong pulsation would then be seen to be associated with a large scale height of the molecular atmosphere.
\end{abstract}

Key words. stars: carbon - stars: AGB and post-AGB - circumstellar matter - stars: mass-loss - Magellanic Clouds - infrared: stars

\section{Introduction}

One of the main contributors of elements such as carbon and nitrogen, and arguably the most important "factory" of cosmic dust, Asymptotic Giant Branch (AGB) stars represent the final evolutionary stages of intermediate-mass stars ( $M_{\text {ZAMS }} \lesssim 1$ to $\sim 8 M_{\odot}$ ), when they lose up to 80 per cent of their mass at rates of $\dot{M} \sim 10^{-6}$ to $10^{-4} M_{\odot} \mathrm{yr}^{-1}$. Aged between $t \sim 30 \mathrm{Myr}$ and $t \gtrsim 10 \mathrm{Gyr}$, AGB stars chemically enrich the Universe on timescales ranging from less than the dynamical timescale of a galaxy to as long as the age of the Universe. AGB mass loss happens as a result of two mechanisms: (1) strong radial pulsations elevate the stellar atmosphere out to a distance where (2) the temperature is sufficiently low $(T \sim 1000 \mathrm{~K})$ and the density is sufficiently high $\left(n_{\mathrm{H}_{2}} \gtrsim 10^{13} \mathrm{~m}^{-3}\right)$ for dust formation to occur. Radiation pressure from the luminous giant $\left(L \sim 10^{4} L_{\odot}\right)$ drives away the dust, taking the gas along with it by mutual collisions. During this "superwind" stage the dust-enshrouded AGB star vanishes at optical wavelengths but shines brightly in the infrared.
An unsolved problem of AGB mass loss is how the simple molecules in the stellar photosphere grow into larger assemblies that form the cores for dust condensation, and how efficient these processes are. Little is known about the atmospheric chemistry, the molecular abundances within the dustformation zone, and the dust-to-gas ratio in the wind. How do molecular chemistry and dust condensation depend on the stellar mass, luminosity, temperature, pulsation period and amplitude, on the initial metallicity and on the self-enrichment of the photosphere as a result of the dredge-up of nucleosynthesis products? We do know that the transformation of some oxygenrich AGB stars into carbon stars (by dredge-up on the upper AGB) changes the molecular and dust formation chemistry from oxygen-dominated to carbon-dominated, giving rise to a vastly different array of molecules and dust particles. Hence these two types of AGB star enrich the interstellar medium with completely different material.

The Large and Small Magellanic Clouds (LMC and SMC) offer an excellent opportunity to study AGB mass loss, at metallicities of $\sim 40$ and 15 per cent solar, respectively. 
Accurate luminosities and mass-loss rates can be obtained in these galaxies as a result of their proximity and known distances of 50 and $60 \mathrm{kpc}$, respectively. Much work on magellanic dust-enshrouded AGB stars has been done (e.g., Wood et al. 1992; Zijlstra et al. 1996; van Loon et al. 1997, 1998). The mass-loss rate depends strongly on luminosity but also on temperature and pulsation properties (Wood 1998; van Loon et al. 1999, 2005; Marshall et al. 2004; Whitelock et al. 2003). Dust mass-loss rates are lower in metal-poor environments, but this may not be true for the gas mass-loss rates (van Loon 2000).

Metal-poor carbon stars are surprisingly abundant in $\mathrm{C}_{2}$ and $\mathrm{C}_{2} \mathrm{H}_{2}$, and may have a higher $\mathrm{C} / \mathrm{O}$ ratio than their galactic solar-metallicity equivalents (van Loon et al. 1999; Matsuura et al. 2002, 2005). This may be due to the high contrast between the carbon self-enrichment and oxygen-poor photosphere of metal-poor carbon stars, and could have important consequences for the condensation of carbonaceous dust grains in low-metallicity environments.

To investigate the formation of simple, di-atomic molecules such as $\mathrm{C}_{2}$ and $\mathrm{CN}$, the assembly into slightly more complex molecules such as $\mathrm{C}_{2} \mathrm{H}_{2}$ and $\mathrm{HCN}$, and the condensation into dust grains, we set out to measure the molecular abundances in the extended atmospheres of mass-losing carbon-rich AGB stars in the LMC. Because many of these highly-evolved objects are dust-enshrouded, it is impossible to perform such a study at optical wavelengths. Furthermore, whilst the optical spectrum is rich in bands of di-atomic molecules, larger molecules are observed at infrared wavelengths. The three micron telluric window $(2.9-4.1 \mu \mathrm{m})$ in carbon stars shows distinctive broad and strong absorption by $\mathrm{C}_{2} \mathrm{H}_{2}$ and $\mathrm{HCN}$ molecules at $3.1 \mu \mathrm{m}$, and often absorption by $\mathrm{C}_{2} \mathrm{H}_{2}$ at $3.8 \mu \mathrm{m}$ and $\mathrm{HCN}$ at $3.57 \mu \mathrm{m}$. These molecules are formed in the upper atmospheric layers by hydration of the di-atomic molecules that are seen at optical wavelengths. $\mathrm{C}_{2} \mathrm{H}_{2}$ is a possible building block for Polycyclic Aromatic Hydrocarbons (PAHs) (Latter 1991), and must play a key rôle in the condensation of carbonaceous grains in H-rich environments (Gail \& Sedlmayr 1988). The HCN band strength may be a measure of the nitrogen abundance.

Here we present ESO/VLT three micron spectra of a large sample of heavily dust-enshrouded carbon stars in the LMC, and an analysis of their molecular atmospheres.

\section{Observations}

\subsection{Targets}

The targets for our 3-4 $\mu \mathrm{m}$ spectroscopy were drawn from a sample of mid-IR sources detected with the Mid-course Space eXperiment (MSX) (Egan et al. 2001; Wood \& Cohen 2001). Our principal objective was to establish the nature and chemical composition of heavily dust-enshrouded stars for which optical spectroscopy would not be possible, to help select targets for our Spitzer Space Telescope programme \#3505 (PI: P. R. Wood). Hence we selected objects by excluding all objects that have optical photometry in the MACHO database, bright objects with a $K_{\mathrm{s}}$-band magnitude $<9$ mag, and objects that are not extremely red with a $J-K_{\mathrm{s}}$ colour $<5$ mag. The actually observed objects are mostly of 8th or 9th magnitude in the 3-4 $\mu \mathrm{m}$ range. Throughout the paper we refer to the MSX-selected sample by their MSX LMC catalogue numbers.

Six more dusty objects were observed that were known to be in or near the populous LMC clusters NGC 1903, NGC 1984 and NGC 1978 (van Loon et al. 2005). VLT/ISAAC spectra covering the same spectral region are available from the literature for nine more IR objects in the LMC (Matsuura et al. 2005). Of these, IRAS 04496-6958, IRAS 04557-6753 and LI-LMC 1813 are cluster members (van Loon et al. 2005b). The IR photometric data of the cluster objects can be found in van Loon et al. (2005b) and are not repeated here.

\subsection{Spectroscopy}

The ISAAC instrument on the European Southern Observatory (ESO) Very Large Telescope (VLT) "Antu" at Paranal, Chile, was used on the nights of 6, 7 and 8 December 2003 to obtain low-resolution long-slit spectra between 2.9 and $4.1 \mu \mathrm{m}$. At a slit width of $2^{\prime \prime}$ the spectral resolving power was essentially determined by the seeing and was $300 \lesssim R \lessgtr 700$. The smallest attained resolution element is sampled by three pixels on the Aladdin array.

The spectra were obtained by chopping and nodding with a throw of $10^{\prime \prime}\left(15^{\prime \prime}\right.$ in some cases to avoid chopping onto another star) to cancel the high background at these thermal-IR wavelengths, and jittering within $2^{\prime \prime}$ to remove the effects of bad pixels. This produced three spectra on the final combined frame, with the central spectrum twice as bright as the other (inverse) spectra. Exposure times varied between 12 and $60 \mathrm{~min}$. After removing cosmic ray hits and correcting the spectra for their small inclination with respect to the array, all spectra were extracted using an optimal extraction algorithm. The extracted spectra were then aligned and combined. Spectra of an internal Xe+Ar lamp were used to calibrate the wavelengths; the spectra are sampled on a grid of $14 \AA$ elements.

The relative spectral response calibration was obtained by dividing by the spectrum of the B-type standard star HIP 020020. This removed most of the telluric absorption lines - except the saturated methane line at $3.32 \mu \mathrm{m}$ - but introduced artificial emission features due to photospheric absorption lines in the spectrum of HIP 020020. Each spectrum was therefore multiplied by a synthetic spectrum appropriate for HIP 020020 , in which we have inserted absorption lines due to $\operatorname{Br} \alpha 4.052$, $\operatorname{Pf} \gamma$ 3.741, $\operatorname{Pf} \delta 3.297, \operatorname{Pf} \epsilon 3.039$ and $\operatorname{Pf} \zeta 2.873 \mu \mathrm{m}$ of depths sufficient to remove the spurious emission lines. Small wavelength shifts and differences in effective resolution can cause imperfections in the correction. Figure 1 shows the observed uncorrected spectra of HIP 020020 and target star MSX LMC 47, a low-resolution Kurucz spectrum $\left(T_{\text {eff }}=18000 \mathrm{~K}, \log g=4.0\right.$ and $[\mathrm{Fe} / \mathrm{H}]=0$ ) and our synthetic spectrum. The observed spectra show the extent to which the telluric absorption affects the spectrum especially around $3.2-3.3 \mu \mathrm{m}$, and the effective band width between 2.85 and $4.15 \mu \mathrm{m}$.

A $\kappa-\sigma$ clipping algorithm was applied to remove spikes from the spectrum that are caused by hot pixels. Care was taken 


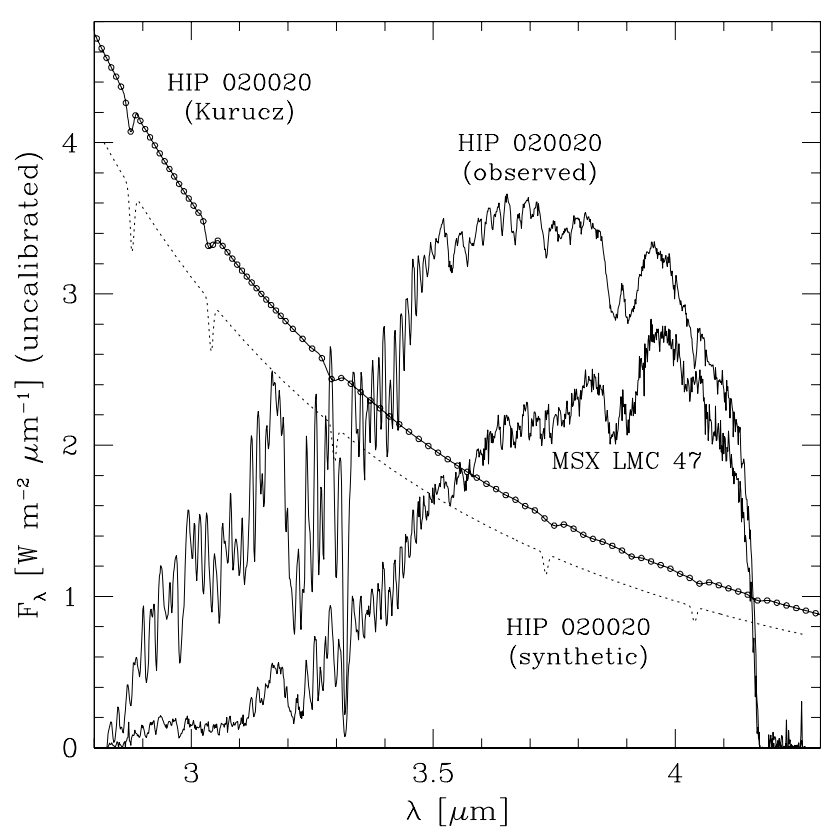

Fig. 1. Uncorrected ESO-VLT/ISAAC spectra of the B-type standard star HIP 020020 and a target star, MSX LMC 47, plus a low-resolution Kurucz spectrum and our synthetic spectrum appropriate for the photosphere of HIP 020020.

to ensure that astrophysical features were not affected by this procedure.

The conditions were very similar for all three nights. The seeing as measured on the acquisition images was around $0.5^{\prime \prime}$ most of the time but occasionally worsened up to about $1^{\prime \prime}$ (e.g., when observing at high airmass of $\sim 2$ ). The relative humidity and air temperature were fairly constant at 8-18 per cent and $12-15^{\circ} \mathrm{C}$, respectively.

\subsection{Photometry}

IR photometry (Table 1) is compiled for all targets and for the Matsuura et al. (2005) objects, except for cluster members (see van Loon et al. 2005b). A few have been monitored at nearIR wavelengths to measure the pulsation period and amplitude (Table 2).

$J K L$-band photometry for the targets was obtained at Mount Stromlo and Siding Springs Observatory (MSSSO) as part of a long-term monitoring campaign (Wood et al., in preparation). In addition, $K_{\mathrm{s}}$-band photometry from the 2-Micron All-Sky Survey (2MASS) is available for almost all targets, but most targets are too faint in the $J$-band and many are even too faint in the $H$-band to have 2MASS data at those wavelengths. Very few targets are detected in the DENIS survey, which is inferior to 2MASS in terms of sensitivity. Only three MSX-selected targets have a DENIS $K_{\mathrm{s}}$-band measurement (Table 2). Additional $L^{\prime}$-band photometry is available from the spectroscopy acquisition images, accurate to within a few $0.1 \mathrm{mag}$.

All prime targets have reliable MSX band A $(8.3 \mu \mathrm{m})$ flux densities listed in the MSX Point Source Catalogue (Version 2.3). We also collected InfraRed Astronomical
Satellite (IRAS) scans from the IRAS data server ${ }^{1}$ and used the Groningen Image Processing SYstem (GIPSY) software with the SCANAID tool to reconstruct a cut through the emission on the exact position. This is superior to the IRAS Point Source Catalogue values and works well for isolated sources for which often reliable flux densities can be estimated down to a level of a few $0.01 \mathrm{Jy}$ at 12 and $25 \mu \mathrm{m}$. A few targets have also been observed with the Infrared Space Observatory (ISO) (Table 2).

\section{Results}

\subsection{IR carbon stars}

The majority (28) of our targets turn out to be carbon stars, on the basis of strong carbonaceous molecular absorption features seen in the spectrum around 3.1 and often $3.8 \mu \mathrm{m}$ (Fig. 2). Out of these, 10 are part of Spitzer Space Telescope GO programme \#3505 (PI: P. Wood).

It is worth noting that MSXLMC 307 has the longest known pulsation period of any carbon star: 889 d (Wood 1998) or $939 \mathrm{~d}$ (Whitelock et al. 2003).

MSXLMC 55 is misidentified in the literature with the M-type giant HV 11977, of which we also took a spectrum.

Another mistaken identity occurs for MSX LMC 45: Leisy et al. (1997) identified the IRAS source with the low-excitation Planetary Nebula SMP LMC 36 (Sanduleak et al. 78), located at $9^{\prime \prime}$ WNW from an extremely red 2MASS source. Our 3-4 $\mu \mathrm{m}$ spectrum is of the 2MASS object, whilst the PN is a target for Spitzer Space Telescope GTO programme \#103 (P.I.: J. Houck). Although the $K_{\mathrm{s}}-L^{\prime}$ colour of SMP LMC 36 (Table 3) is very red at $2.76 \mathrm{mag}$, MSX LMC 45 is much redder at 4.95 mag and with $L^{\prime}=7.87$ mag much brighter in the thermal IR than SMP LMC 36 which has $L^{\prime}=11.24$ mag. For all effects the emission detected at 8.3 (MSX), 12 and $25 \mu \mathrm{m}$ (IRAS) arises from the carbon star.

\subsection{IR objects of unknown type}

For two of our targets, the $3-4 \mu \mathrm{m}$ spectrum does not lead to an unambiguous photospheric chemical classification (Fig. 3). Coincidentally, these are the only two objects in our target sample that are part of Spitzer Space Telescope programmes other than GO \#3505. Three more objects turned out to be massive Young Stellar Objects; these are analysed in detail in van Loon et al. (2005c) and Oliveira et al. (in preparation).

The 3-4 $\mu \mathrm{m}$ spectrum of MSX LMC 196 displays a hint of absorption around 3.0-3.1 and 3.7-3.8 $\mu \mathrm{m}$, in which case it is a carbon star. The molecular features may be heavily veiled by the strong dust continuum emission. Indeed, with $F_{12}=F_{25}$ its [12]-[25] colour is redder than any of the IR carbon stars.

On the basis of the IR colours, van Loon et al. (1998) classified the dust around MSX LMC 733 as oxygen-rich, but in a re-analysis including ISO data Trams et al. (1999) classified it as carbon-rich. They also present a CAM-CVF spectrum, in which broad emission around $11.3 \mu \mathrm{m}$ was interpreted as $\mathrm{SiC}$, which would confirm its carbon-rich nature. However,

\footnotetext{
${ }^{1}$ http://www . astro.rug.nl/IRAS-Server/
} 
Table 1. List of targets, in order of increasing Right Ascension (2MASS coordinates, in J2000). MSX LMC (Egan et al. 2001; targets for Spitzer Space Telescope programmes are in boldface) and IRAS PSC designations are given where available, together with near-IR magnitudes $(1=$ 2MASS, 2 = MSSSO, 3 = spectroscopy acquisition images) and MSX band-A and IRAS 12 and $25 \mu \mathrm{m}$ flux densities (in mJy). Values marked with a colon are suspect. References to the identification as a dust-enshrouded AGB star are as follows: $1=$ Egan et al. $(2001) ; 2=$ Loup et al. (1997); 3 = Reid et al. (1990); 4 = Trams et al. (1999); 5 = van Loon et al. (1997); 6 = van Loon (2000); $7=$ Zijlstra et al. (1996); $8=$ this work.

\begin{tabular}{|c|c|c|c|c|c|c|c|c|c|c|c|c|c|c|}
\hline MSX & IRAS & $\alpha\left(\mathrm{h} \mathrm{m} \mathrm{s}^{2}\right)$ & $\delta\left({ }^{\circ}, \prime \prime\right)$ & $J(1)$ & $H(1)$ & $K_{\mathrm{s}}(1)$ & $J(2)$ & $K(2)$ & $L(2)$ & $L^{\prime}(3)$ & $F_{\mathrm{A}}$ & $F_{12}$ & $F_{25}$ & Ref \\
\hline \multicolumn{15}{|c|}{ IR carbon stars } \\
\hline 1042 & $04374-6831$ & 43722.7 & -682503 & 16.33 & 13.76 & 11.55 & & & & 9.31 & 107 & 240 & 120 & 2,7 \\
\hline 1249 & $04552-6536$ & 45527.6 & -653107 & 14.15 & 12.27 & 10.77 & & & & 8.18 & 216 & 160 & 110 & 2,5 \\
\hline 50 & 05026-6809 & 50222.0 & -680524 & $>17.9$ & $>16.1$ & 14.67 & & 12.44 & & 8.78 & 194 & $250:$ & 200: & 2,5 \\
\hline 91 & $05038-6857$ & 50338.5 & -685313 & $>16.4$ & 15.56 & 12.87 & 16.67 & 11.25 & & 9.24 & 238 & 420 & 250 & 1 \\
\hline 93 & & 50527.9 & -685636 & 16.83 & 14.36 & 12.01 & & 13.01 & 9.92 & 8.82 & 170 & 160 & 100 & 1 \\
\hline 55 & $05058-6843$ & 50541.7 & -683911 & & & & & 11.24 & & 9.25 & 161 & 260 & 170 & 8 \\
\hline 45 & $05108-6839$ & 51041.3 & -683607 & $>16.3$ & 14.56 & 11.74 & & 12.82 & & 7.87 & 372 & 430 & 380 & 1 \\
\hline $47^{d}$ & 05113-6739 & 51113.9 & -673616 & $>17.7$ & 14.77 & 12.49 & & & & 8.02 & 233 & 250 & 140 & 7 \\
\hline $219^{d}$ & & 51119.5 & -684228 & $>15.6$ & $>14.7$ & 13.14 & 17.93 & 12.31 & & 8.62 & 204 & 180 & 120 & 1 \\
\hline 232 & & 51204.0 & -691621 & $>16.9$ & 14.93 & 12.84 & 17.78 & 12.52 & 9.73 & 9.23 & 135 & 220 & 120 & 1 \\
\hline $\mathbf{2 2 3}^{d}$ & $05132-6941$ & 51251.1 & -693750 & $>17.8$ & $>15.7$ & 13.36 & & 13.00 & 9.34 & 8.33 & 259 & 220 & 130 & 1 \\
\hline 202 & & 51414.9 & -700410 & $>18.1$ & 15.92 & 13.07 & 17.81 & 12.36 & & 9.76 & 176 & 120 & 70 & 1 \\
\hline 225 & & 51437.9 & -681921 & $>18.0$ & $>16.7$ & 13.42 & & 12.73 & & 8.48 & 215 & 200 & 140 & 1 \\
\hline 221 & & 51515.7 & -690034 & $>16.1$ & 14.92 & 12.74 & 17.36 & 11.93 & 9.37 & 9.10 & 189 & 260 & 220 & 1 \\
\hline $349^{d}$ & & 51726.9 & -685458 & $>17.4$ & $>16.9$ & 14.82 & & 13.49 & 9.81 & 9.04 & 193 & 220 & 130 & 1 \\
\hline $\mathbf{3 0 7}^{d}$ & $05190-6748$ & 51856.3 & -674504 & $>18.2$ & $>15.9$ & 13.10 & & & & 8.62 & 209 & 390 & 250 & 3 \\
\hline $\mathbf{3 4 1}^{d}$ & & 52100.4 & -692055 & $>17.5$ & 15.92 & 13.15 & 17.47 & 12.08 & 9.44 & 9.59 & 210 & 100 & 60 & 1 \\
\hline 484 & & 52503.3 & -692617 & $>15.2$ & $>14.6$ & 14.48 & & 13.77 & 10.59 & 9.43 & 156 & $<100$ & $<100$ & 1 \\
\hline 774 & & 52623.1 & -691120 & $>16.8$ & 15.08 & 12.72 & 18.00 & 12.49 & 9.54 & 8.87 & 237 & $<250$ & 140: & 1 \\
\hline 677 & & 52711.0 & -693414 & $>16.2$ & 14.01 & 11.90 & 16.99 & 11.69 & 9.18 & 8.82 & 105 & 180 & 90 & 1 \\
\hline $635^{d}$ & $05278-6942$ & 52724.1 & -693945 & $>17.5$ & $>15.6$ & 12.35 & 18.13 & 11.98 & 8.45 & 9.60 & 684 & 450 & 380 & 2,5 \\
\hline $692^{d}$ & $05295-7121$ & 52846.6 & -711913 & 16.80 & 14.32 & 12.18 & & & & 8.67 & 204 & 230 & 80 & 2,7 \\
\hline 644 & & 52922.9 & -700646 & $>16.4$ & 14.28 & 11.93 & 17.46 & 11.92 & 8.72 & 8.34 & 232 & 160 & 100 & 1 \\
\hline 654 & & 53005.1 & -695646 & $>14.4$ & $>13.8$ & 13.10 & & 12.39 & 9.36 & 9.81 & 152 & 200: & 100: & 1 \\
\hline 1780 & & 53301.8 & -682359 & $>17.9$ & $>17.0$ & 14.10 & & 13.76 & & 8.74 & 309 & 400 & $<600$ & 1 \\
\hline $\mathbf{7 4 3}^{d}$ & & 53453.7 & -702925 & $>14.1$ & $>13.2$ & 13.27 & 18.65 & 12.82 & 9.67 & 9.37 & 165 & 260 & 100: & 1 \\
\hline $872^{d}$ & $05360-6648$ & 53601.2 & -664640 & $>18.0$ & 16.07 & 13.28 & & & & 8.64 & 192 & 210 & 90 & 3 \\
\hline 737 & & 53636.8 & -704346 & 16.58 & 13.72 & 11.42 & 16.32 & 11.27 & & 8.31 & 153 & 310 & 150: & 1 \\
\hline \multicolumn{15}{|c|}{ IR objects of unknown type } \\
\hline $196^{b}$ & $05125-7035$ & 51200.8 & -703224 & $>18.0$ & $>16.8$ & 14.52 & & & & 10.59 & 451 & 500 & 500 & 2,5 \\
\hline $\mathbf{7 3 3}^{a}$ & $05348-7024$ & 53416.0 & -702253 & $>17.7$ & 15.49 & 12.85 & & 12.70 & 9.28 & 9.44 & 414 & 580 & 160 & 2,7 \\
\hline \multicolumn{15}{|c|}{ LMC carbon-rich stars from Matsuura et al. (2005) } \\
\hline $1007^{c}$ & $04286-6937$ & 42830.2 & -693050 & 16.35 & 13.87 & 11.86 & & & $8.84^{A}$ & & 155 & 280 & 200 & 2,7 \\
\hline 1198 & $04539-6821$ & 45346.4 & -681613 & $>18.1$ & 15.44 & 13.03 & & & $9.50^{A}$ & & 193 & 220 & 120 & 2,7 \\
\hline $\mathbf{4 4}^{d}$ & $05112-6755$ & 51110.5 & -675211 & 16.41 & 14.07 & 11.69 & & & $9.13^{A}$ & & 451 & 460 & 330 & 3 \\
\hline$-^{B}$ & & 52046.7 & -690124 & 11.43 & 10.45 & 9.77 & & & $8.80^{A}$ & & 65 & 60: & 20: & 4 \\
\hline \multicolumn{15}{|c|}{ LMC oxygen-rich stars from Matsuura et al. (2005) } \\
\hline $\mathbf{2 8 3}^{a}$ & $05128-6455$ & 51304.6 & -645140 & 14.55 & 12.83 & 11.28 & & & $9.00^{A}$ & & 259 & 230 & 240 & 2,7 \\
\hline $264^{c}$ & $05148-6730^{C}$ & 51449.7 & -672720 & 8.64 & 7.78 & 7.42 & & & $6.97^{A}$ & & 331 & 440 & 230 & 3 \\
\hline
\end{tabular}

Spitzer programmes: ${ }^{a}$ GTO \#200 (Houck); ${ }^{b}$ DDT \#1094 (Kemper); ${ }^{c}$ GO \#3426 (Kastner); ${ }^{d}$ GO \#3505 (Wood). Notes: ${ }^{A}$ SAAO monitoring at $3.5 \mu \mathrm{m}$ (Whitelock et al. 2003); ${ }^{B}$ BMB-R 46, SHV 0521050-690415; ${ }^{C}$ HV 916.

its CAM-CVF spectrum is not unlike that of OH/IR stars 3-4 $\mu$ m spectrum of MSX LMC 733 is similar to the ISO-SWS where the silicate feature is seen in self-absorption. Overall the spectrum of the M8 giant CD Gru (Vandenbussche et al. 2002). 
Table 2. Additional data from DENIS and ISO, and pulsation properties from near-IR monitoring. The flux densities are in mJy, pulsation periods in days and $K_{\mathrm{s}}$-band amplitudes in magnitudes. The references are as follows: $1=$ Loup et al. (in preparation); $2=$ Trams et al. (1999); 3 = van Loon et al. (2005a); 4 = van Loon et al. (2005b); $5=$ Whitelock et al. (2003); 6 = Wood (1998).

\begin{tabular}{|c|c|c|c|c|c|c|c|c|c|c|c|c|}
\hline Name & $I_{\text {DENIS }}$ & $J_{\text {DENIS }}$ & $K_{\mathrm{s}, \mathrm{DENIS}}$ & $F_{4.5}$ & $F_{6.7}$ & $F_{12}$ & $F_{14.3}$ & $F_{25}$ & Spectrum & $P(\mathrm{~d})$ & $\Delta K_{\mathrm{s}}$ & Ref. \\
\hline \multicolumn{13}{|l|}{ IR carbon stars } \\
\hline MSX LMC 1042 & & & & & & 185 & & 86 & PHOT-S & 639 & 1.44 & 2,5 \\
\hline MSX LMC 1249 & 17.57: & 14.49 & 10.60 & & & & & & & & & \\
\hline MSX LMC 93 & & & 12.13 & & & & & & & & & \\
\hline MSX LMC 47 & & & & & & 260 & & 67 & & 707 & 1.79 & $2,5,6$ \\
\hline MSX LMC 307 & & & & & & 346 & & 163 & PHOT-S & 914 & 1.74 & $2,5,6$ \\
\hline MSX LMC 341 & & & & 7 & & 8 & & & & & & 1 \\
\hline MSX LMC 692 & & 14.75 & 10.46 & & & 143 & & 7: & & 682 & 1.15 & 2,5 \\
\hline MSX LMC 644 & & & & 127 & & 222 & & & & & & 1 \\
\hline MSX LMC 654 & & & & 118 & 138 & 155 & 135 & & & & & 4 \\
\hline MSX LMC 872 & & & & & & 171 & & 82 & & 534 & 1.26 & $2,5,6$ \\
\hline \multicolumn{13}{|c|}{ IR objects of unknown type } \\
\hline MSX LMC 733 & & & & & & 525 & & 208 & CAM-CVF & & & 2 \\
\hline \multicolumn{13}{|c|}{ LMC carbon-rich stars from Matsuura et al. (2005) } \\
\hline IRAS 04286-6937 & 17.58: & 15.37 & 10.95 & & & 136 & & 59 & & 662 & 1.13 & 2,5 \\
\hline IRAS 04539-6821 & & & & & & 244 & & 89 & & 676 & 1.65 & 2,5 \\
\hline IRAS 05112-6755 & & & 11.65 & & & 387 & & 108 & PHOT-S & 826 & 1.72 & $2,5,6$ \\
\hline BMB-R 46 & 14.33 & 11.72 & 9.42 & 67 & & 51 & & & & 541 & 0.65 & $1,2,5$ \\
\hline \multicolumn{13}{|c|}{ LMC oxygen-rich stars from Matsuura et al. (2005) } \\
\hline IRAS $05128-6455$ & 17.87 & 13.43 & & & & 226 & & 61 & PHOT-S & 409 & 0.30 & $2,3,5$ \\
\hline IRAS $05148-6730$ & 10.47 & & 7.34 & & & 380 & & 176 & & 951 & 0.24 & $2,3,5$ \\
\hline
\end{tabular}

\subsection{Serendipitous objects}

In several cases a spectrum could be taken of another IR-bright object, simultaneously with the principal target. These "serendipitous" objects (Fig. 4 and Table 3) are not generally dust-enshrouded.

The M 4 supergiant HV 2532 is responsible for most of the mid-IR emission associated with IRAS 05267-6913 $\left(F_{12} \sim\right.$ $\left.0.20 \mathrm{Jy}, F_{25} \sim 0.25 \mathrm{Jy}\right)$. The MSX data clearly separates its emission, MSXLMC $592\left(F_{\mathrm{A}}=230 \mathrm{mJy}\right)$, from that of our spectroscopy target, MSX LMC 774.

\subsection{Cluster IR objects}

We took spectra of six cluster IR objects from van Loon et al. (2005b) (Fig. 5). Four of these, NGC 1903-IR1 and IR2 and NGC 1978-IR1 and IR2 are carbon stars. The other two, NGC 1984-IR1 and IR2 are oxygen-rich objects; IR1 (=IRAS 05280-6910) is a source of OH (Wood et al. 1992; Marshall et al. 2004) and $\mathrm{H}_{2} \mathrm{O}$ maser emission (van Loon et al. 2001) whilst IR2 is the M1 supergiant WOH G347.

Two objects in our MSX-selected target list, MSXLMC 484 and 654 , and three of the carbon stars with 3-4 $\mu \mathrm{m}$ spectra published in Matsuura et al. (2005), IRAS 04496-6958, IRAS 04557-6753 and LI-LMC 1813 were also found to be associated with clusters (van Loon et al. 2003; van Loon et al. 2005b).

\section{Analysis}

\subsection{Luminosities and mass-loss rates}

As a result of the selection criteria, the MSX-selected targets have very red $K_{\mathrm{s}}-L^{\prime}$ (Fig. 6). This is caused by circumstellar extinction diminishing the $K_{\mathrm{s}}$-band brightness and circumstellar dust emission enhancing the $L^{\prime}$-band brightness. Hence their faint $K_{\mathrm{s}}$-band brightness is not a direct consequence of their bolometric luminosity. IR carbon stars are objects which are known to be variable with amplitudes up to $2 \mathrm{mag}$ at near-IR wavelengths (e.g., Wood 1998; Whitelock et al. 2003). Although the 2MASS, MSX and IRAS data refer to combined measurements taken at different epochs, the groundbased nearIR data that we use here are single-epoch measurements.

The spectral energy distributions of the IR carbon stars were modelled with the radiative transfer code DUSTY (Ivezić et al. 1999). The density distribution is based upon a hydrodynamic computation of a dust-driven wind at constant mass-loss rate. The model was then scaled to match the overall observed Spectral Energy Distribution (SED), knowing the distance to the LMC $(50 \mathrm{kpc})$, which then yields an accurate measurement of the bolometric luminosity.

A blackbody was used to represent the underlying stellar radiation field, with a temperature of $T_{\text {eff }}=2500 \mathrm{~K}$. We used $0.1 \mu \mathrm{m}$ grains of amorphous carbon dust (Henning \& Mutschke 1997). To obtain the mass-loss rate one has to know the dust grain density, for which we adopt $\rho_{\text {grain }}=3 \mathrm{~g} \mathrm{~cm}^{-3}$, and the gas-to-dust mass ratio, for which we adopt $\rho_{\text {gas }} / \rho_{\text {dust }}=500$ at the metallicity of the LMC (two to three times below solar). The results are summarised in Table 4 . 


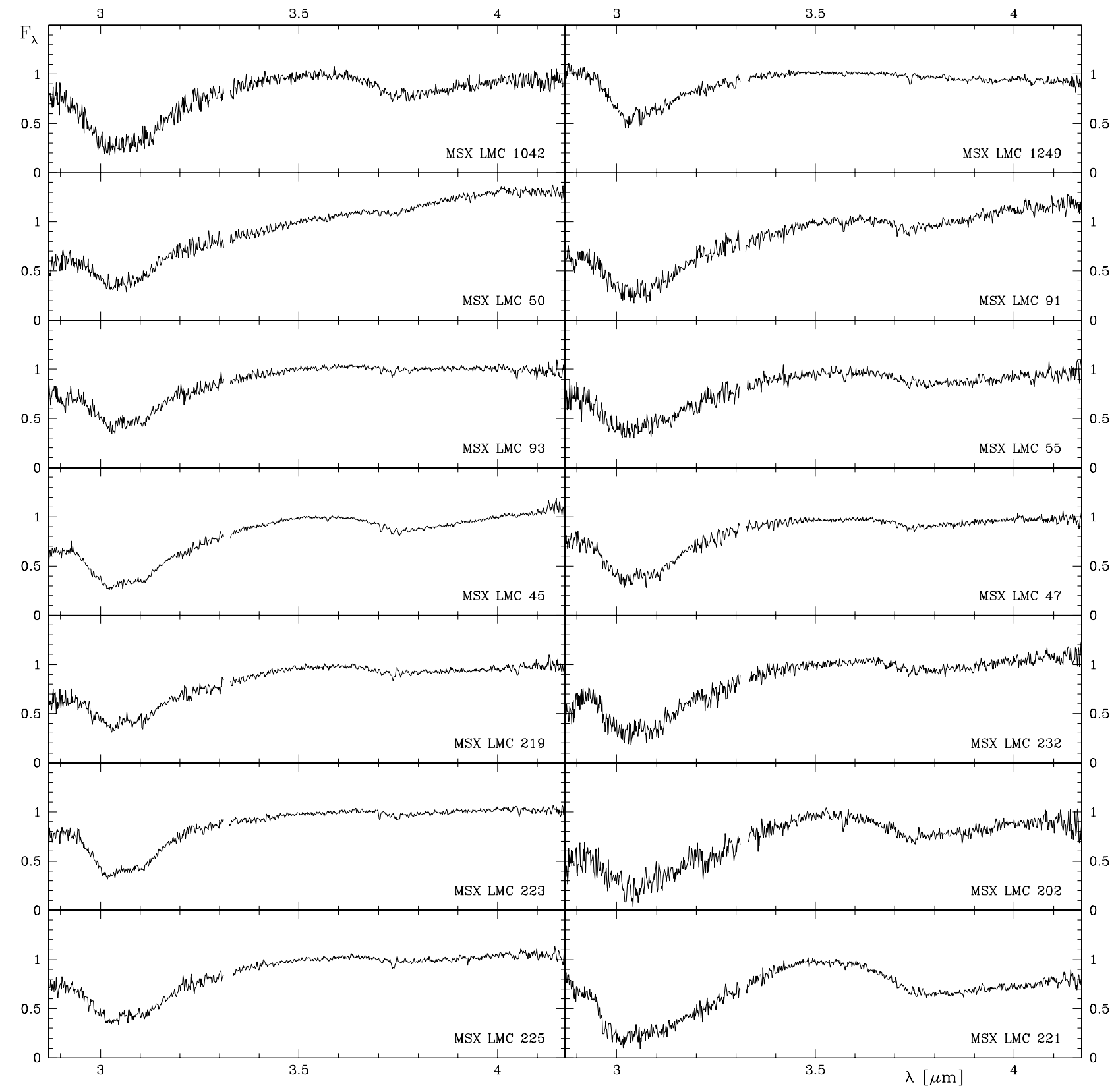

Fig. 2. ESO/VLT 3-4 $\mu \mathrm{m}$ spectra of MSX-selected IR carbon stars. The spectra have been normalised to $F_{3.5}=1$.

We usually found that a satisfactory match between the model and the observed SED required a rather low temperature for the dust at the inner edge of the envelope, $T_{\text {dust }} \sim 600$ to $700 \mathrm{~K}$. The derived bolometric luminosity and mass-loss rate do not however depend sensitively on the inner-edge dust temperature. The estimated internal accuracies of the luminosity and mass-loss rate are about 5 and 10 per cent, respectively.

The mass-loss rates are correlated with the bolometric luminosities (Fig. 7). Most stars straddle the classical limit to the mass-loss rate for single scattering (long-dashed line in Fig. 7; van Loon et al. 1999b). The observed sequence is steeper than the classical limit though, with luminous stars experiencing mass-loss rates that are higher than this limit - but not as high as the maximum mass-loss rates observed in the LMC (short-dashed lines in Fig. 7; van Loon et al. 1999b).
The two carbon stars with particularly long pulsation periods, $P>800 \mathrm{~d}$, and amplitudes, $\Delta K_{\mathrm{s}}>1.7 \mathrm{mag}$, MSX LMC 307 and IRAS 05112-6755 (Table 2) are amongst the stars with the highest mass-loss rates and luminosities.

On the whole the mass-loss rates of the IR carbon stars are higher than those of optically bright carbon stars (van Loon et al. 2005a), but amongst the IR carbon stars themselves are stars that have relatively low mass-loss rates for their high luminosity. Four of the carbon stars from van Loon et al. (2005b) and Matsuura et al. (2005) do not appear in Fig. 7 as they have significantly lower mass-loss rates than the stars in the MSX-selected sample. These stars may be in a slightly different evolutionary phase, for instance if they have not yet entered the superwind stage or if they pass through a more quiet episode in their thermal-pulsing cycle. Differences in mass may also 


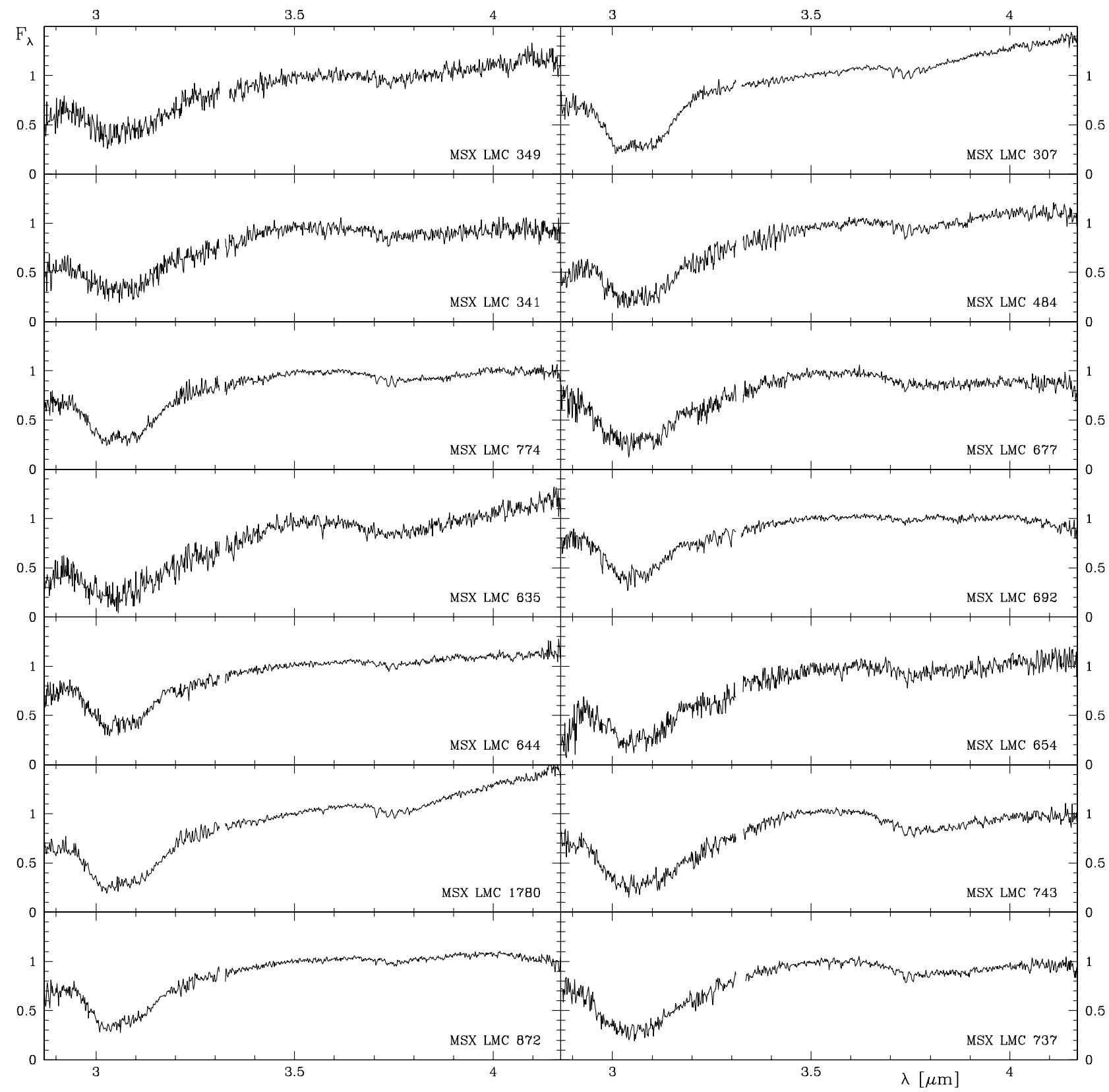

Fig. 2. continued.

contribute to a spread in the mass-loss rate-luminosity plane see van Loon et al. (2005b) for a discussion of the mass-loss rates and luminosities of cluster IR objects in the Magellanic Clouds. The by far most luminous carbon star that we analyse here, IRAS 04496-6958 also lies beyond the boundaries of the diagram. Its mass-loss rate is fairly modest for its high luminosity. In this case there are strong indications for a relatively massive progenitor star $\left(M_{\text {initial }} \sim 5 M_{\odot}\right)$ that may have experienced a thermal pulse recently (van Loon et al. 2005b). For the purpose of the spectral analysis, we divide the sample of IR carbon stars into four groups on the basis of their mass-loss rate and luminosity (Fig. 7 and Table 4).

Stars with high mass-loss rates have a red $K_{\mathrm{s}}-L^{\prime}$ colour or, if having a relatively modestly red $K_{\mathrm{S}}-L^{\prime}$ colour they have a red $L^{\prime}-$ [8.3] colour (Fig. 8). The fact that the $L^{\prime}-$ [8.3] colour shows some correlation with the $K_{\mathrm{S}}-L^{\prime}$ colour but with a large scatter implies that besides the optical depth of the dust shell other parameters play a rôle. These may include the dust temperature at the inner edge of the envelope and perhaps the dust type, or photometric variability.

\subsection{Identification of spectral features}

A grand average of all spectra of the IR carbon stars (Fig. 9) shows all the spectral features of interest at a much reduced noise level. Examples of four types of spectral appearance are given in Fig. 10, to show the similarities and differences amongst the IR carbon stars.

Hydrogen absorption lines of the Brackett and Pfund series are seen in many of the spectra, but this may be due to residuals 
Table 3. Serendipitous objects, listed in order of increasing Right Ascension (2MASS coordinates, in J2000), together with near-IR magnitudes from 2MASS $\left(J H K_{\mathrm{s}}\right)$ and the spectroscopy acquisition images $\left(L^{\prime}\right)$. The PN candidate and Spitzer Space Telescope target SMPLMC 36 is included as we obtained $L^{\prime}$-band photometry for it, although no spectrum was taken.

\begin{tabular}{|c|c|c|c|c|c|c|c|}
\hline Name & $\alpha\left(\mathrm{h} \mathrm{m} \mathrm{s}^{2}\right)$ & $\delta\left({ }^{\circ}{ }^{\prime \prime \prime}\right)$ & $J$ & $H$ & $K_{\mathrm{s}}$ & $L^{\prime}$ & Type \\
\hline MSX LMC 50 B & 50216.9 & -680524 & 11.25 & 10.40 & 10.17 & 9.87 & $?$ \\
\hline HV 11977 & 50545.9 & -683854 & 10.57 & 9.77 & 9.53 & 9.24 & $M$ giant \\
\hline SMP LMC 36 ${ }^{a}$ & 51039.7 & -683605 & 16.25 & 15.61 & 14.00 & 11.24 & Planetary Nebula? \\
\hline MSX LMC 349 B & 51724.4 & -685506 & 11.62 & 10.50 & 9.83 & 9.98 & Carbon star \\
\hline HV 2532 & 52627.4 & -691056 & 8.93 & 8.08 & 7.71 & 7.42 & M 4 supergiant \\
\hline MSX LMC 1780 B & 53259.0 & -682351 & 11.10 & 10.54 & 10.46 & 10.12 & Early-type star? \\
\hline
\end{tabular}

Spitzer programme: ${ }^{a}$ GTO \#103 (P.I.: J. Houck).

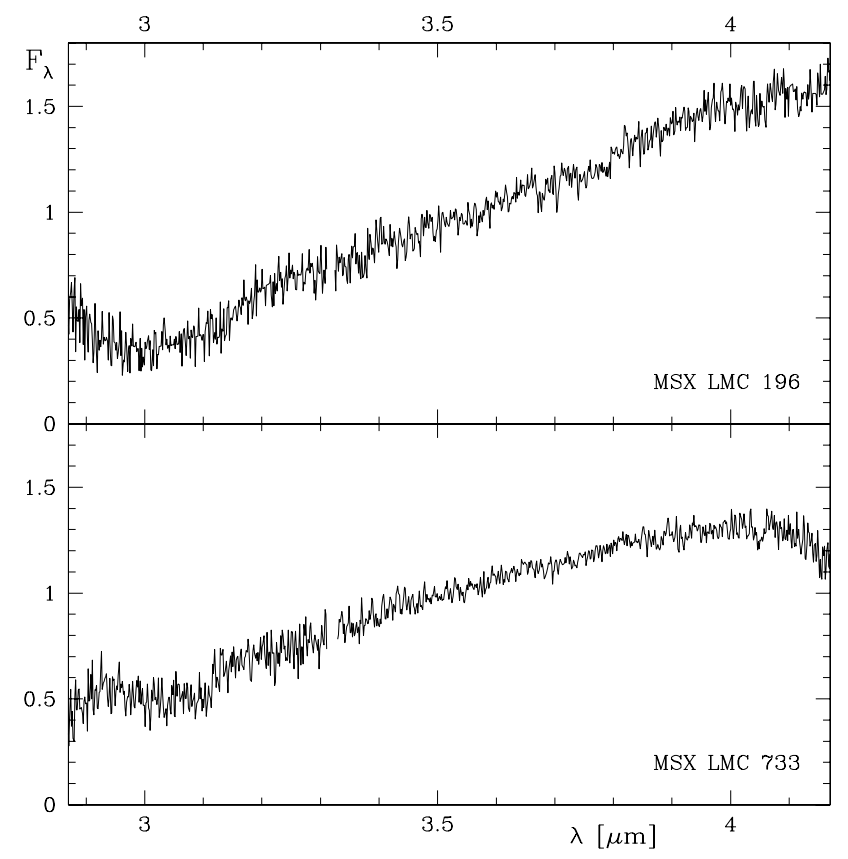

Fig. 3. ESO/VLT 3-4 $\mu \mathrm{m}$ spectra of IR objects of unknown type. MSXLMC 196 shows a hint of absorption around $3.1 \mu \mathrm{m}$ and $3.65-3.80 \mu \mathrm{m}$, and may thus be a heavily veiled carbon star. The overall shape of the spectrum of MSXLMC 733 resembles that of cool oxygen-rich stars better.

in the correction for the same lines in the telluric standard star spectrum. There is no evidence in any of the carbon stars for strong hydrogen line emission arising from shocks in their pulsating atmospheres. A few spectra show an array artifact causing a sharp feature in the extracted spectrum around $3.99 \mu \mathrm{m}$.

The strongest absorption band, between about 2.95 and $3.15 \mu \mathrm{m}$, is seen in all IR carbon stars, and is due to a blend of $\mathrm{HCN}$ and $\mathrm{C}_{2} \mathrm{H}_{2}$. The shape is not universal, although often with characteristic peaks at 3.01 and $3.11 \mu \mathrm{m}$ it sometimes has a rather triangular shape peaking at $3.01 \mu \mathrm{m}$ only (e.g., MSX LMC 1249). Often, the absorption seems to extend to as far as $3.5 \mu \mathrm{m}$ (e.g., MSX LMC 221), but it is not certain that this is still part of the same band. Alternatively, the HNC isomer of $\mathrm{HCN}$ is expected to contribute in precisely this spectral region (Harris et al. 2003).

Most IR carbon stars exhibit a broad depression in the spectrum around $3.8 \mu \mathrm{m}$. In the LMC, the absorption almost always

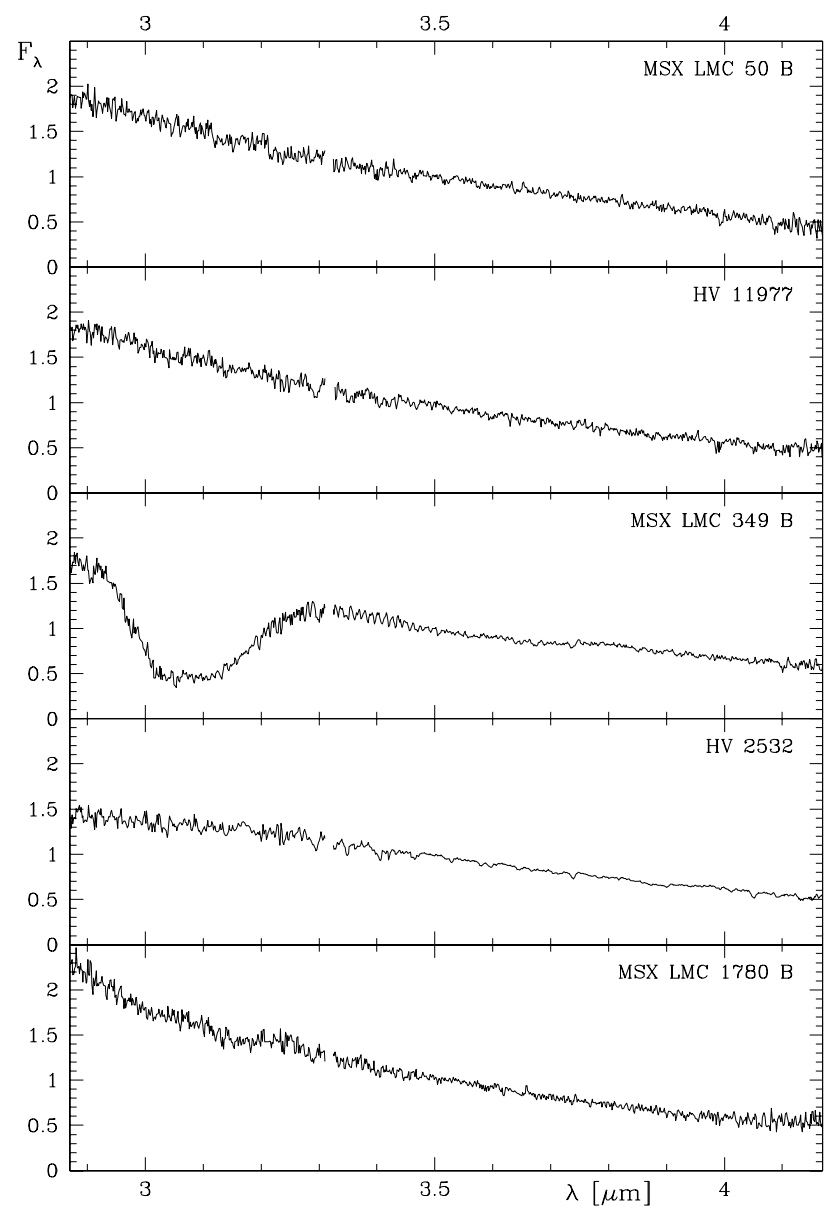

Fig. 4. Serendipitous ESO/VLT 3-4 $\mu$ m spectra (see Table 3). They are all oxygen-rich except MSX LMC 349 B which is an optically bright (yet anonymous) carbon star.

peaks around $3.75 \mu \mathrm{m}$, whereas in galactic carbon stars absorption is normally seen around $3.9 \mu \mathrm{m}$ instead. In our sample, only the less extreme object NGC 1903-IR1 shows evidence for distinct absorption at $3.9 \mu \mathrm{m}$. $\mathrm{CH}$ was suggested to explain a series of absorption lines around $3.75 \mu \mathrm{m}$ in the spectra of the galactic carbon stars TX Psc and WZ Cas (Aoki et al. 1998), but the $\mathrm{CH}$ does not lead to a broad absorption band. Instead, the identification with $\mathrm{C}_{2} \mathrm{H}_{2}$ by Cernicharo et al. (1999) matches the broad absorption seen around $3.75 \mu \mathrm{m}$ in the galactic dustenshrouded carbon star IRC +10216 . $\mathrm{HCN}$ is held responsible 


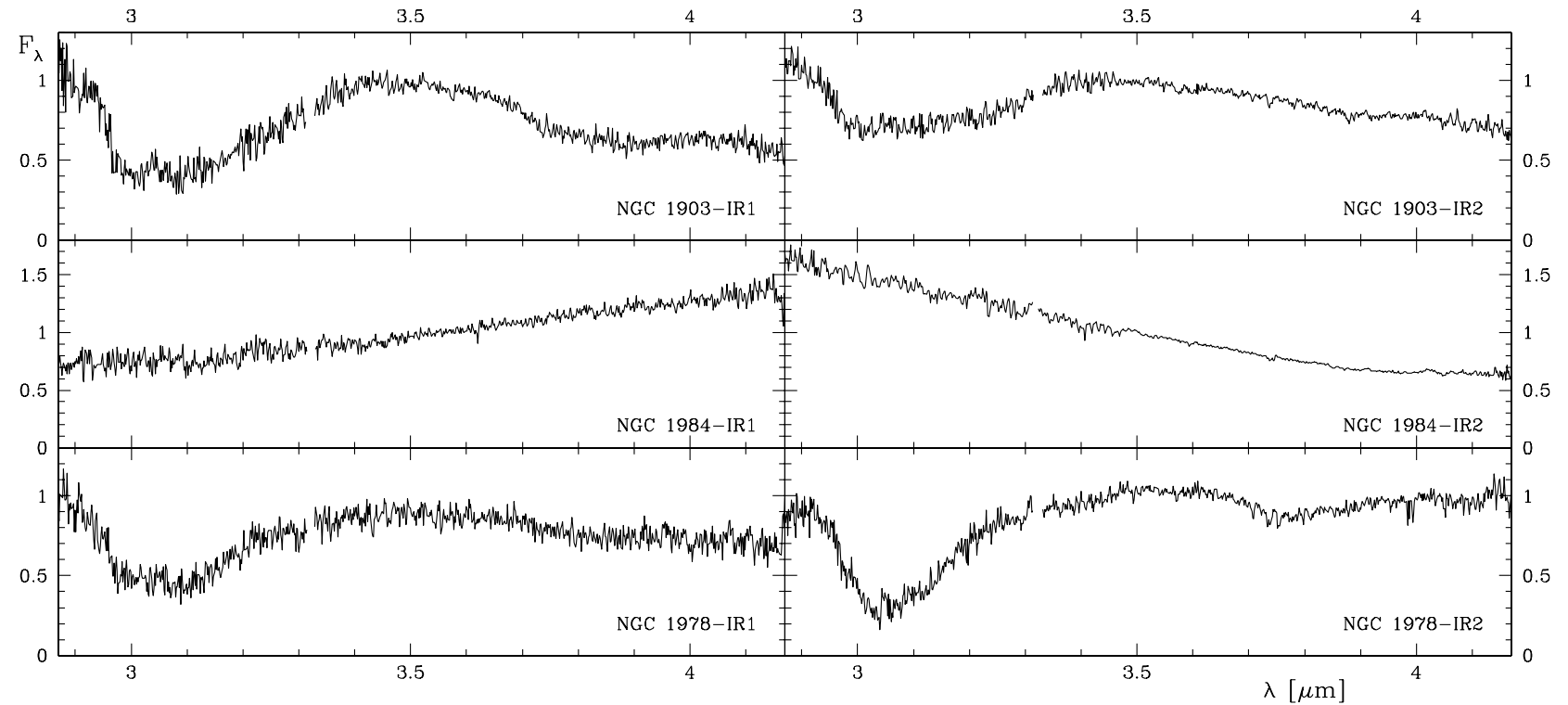

Fig. 5. ESO/VLT 3-4 $\mu \mathrm{m}$ spectra of additional objects in or near populous star clusters (van Loon et al. 2005b). They are all carbon stars except NGC 1984-IR1 and -IR2 which are an OH/IR star and an optically bright red supergiant, respectively.

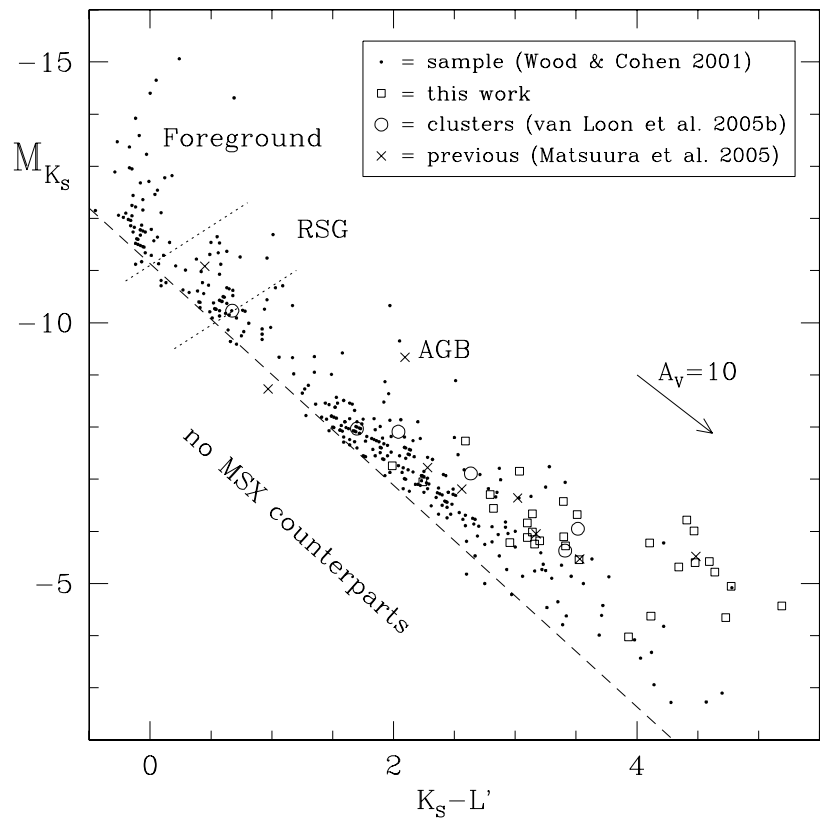

Fig. 6. Absolute $K_{\mathrm{s}}$ brightness versus $K_{\mathrm{s}}-L^{\prime}$ colour-magnitude diagram of the MSX sample, where the objects for which we present spectra (Table 1) are indicated by squares and circles.

for the $3.9 \mu$ m component (Aoki et al. 1998; Harris et al. 2003), but CS may also contribute (Aoki et al. 1998).

A few quite narrow absorption features are seen, superimposed onto the $3.8 \mu \mathrm{m}$ absorption band (Fig. 9). The $3.70 \mu \mathrm{m}$ feature is the most conspicuous of these, but it is always seen in conjunction with a similar feature at $3.76 \mu \mathrm{m}$. A weaker feature at $3.78 \mu \mathrm{m}$ may be related, and there seems to be another feature that is however blended with the Pf $\gamma$ line at $3.74 \mu \mathrm{m}$. Aoki et al. (1998) interpret 3.70 and $3.77 \mu \mathrm{m}$ features as being part of the $\mathrm{CH}(3-2)$ fundamental band, but the stronger associated 3.64 and $3.67 \mu \mathrm{m}$ features (cf.
Jørgensen et al. 1996) are not seen in our spectra (whilst they are seen in TX Psc and WZ Cas) - except perhaps in the enigmatic object MSXLMC 196 (Fig. 3). The features might be part of the $\mathrm{C}_{2} \mathrm{H}_{2}$ band instead (Cernicharo et al. 1999).

$\mathrm{CH}(1-0)$ may be responsible for the series of narrow absorption lines seen around 3.3-3.5 $\mu \mathrm{m}$ in the spectrum of MSX LMC 349 B (Fig. 4). The spectrum of this optically bright carbon star is in fact slightly depressed up to a wavelength of about $3.75 \mu \mathrm{m}$.

A comparison between the average spectra for the four groups in terms of mass-loss rate and luminosity (Fig. 11) fails to reveal any striking correlation of the spectral features with either of these parameters. The dispersion within each group average is quite large, but the error in the mean difference between the group average and the grand average indicates statistically significant differences between the groups. The most obvious difference is in the slope of the spectral continuum, which depends on the optical depth of the circumstellar dust envelope: the continuum is redder at higher mass-loss rates, but at the same mass-loss rate it is redder at lower luminosity.

\subsection{Molecular band strengths}

To investigate the band strengths of some of the spectral features, we compute equivalent widths (Tables 5 and 6). For the narrow 3.57 and $3.70 \mu \mathrm{m}$ bands it is important to realise that the band definitions are appropriate for our spectra and hence include the velocity shift of the LMC $(0.003-0.004 \mu \mathrm{m})$ and the internal wavelength calibration accuracy $( \pm 0.002 \mu \mathrm{m})$. In other data sets these definitions might therefore need a slight readjustment. We recomputed the equivalent widths at 3.1,3.57 and $3.8 \mu \mathrm{m}$ in the spectra from Matsuura et al. (2005) after converting their $F_{v}$ units to our $F_{\lambda}$ units. Different units cause different spectral slopes and hence different continuum definitions, which affects the equivalent width measurements. 
Table 4. Bolometric luminosities, mass-loss rates, inner-edge dust temperatures and optical depth of the IR carbon stars. They are grouped (last column) as follows: $1=$ high $\dot{M}$ and $L ; 2=$ moderate $\dot{M}$ and $L ; 3=$ low $\dot{M}$ and $L ; 4=$ low $\dot{M}$ but high $L$.

\begin{tabular}{|c|c|c|c|c|c|}
\hline Name & $\begin{array}{c}\log (L) \\
\left(L_{\odot}\right)\end{array}$ & $\begin{array}{r}\log (\dot{M}) \\
\left(M_{\odot} \mathrm{yr}^{-1}\right)\end{array}$ & $\begin{array}{l}T_{\text {dust }} \\
(\mathrm{K})\end{array}$ & $\tau_{\mathrm{V}}$ & $\overline{\bar{G}}$ \\
\hline \multicolumn{6}{|l|}{ IR carbon stars } \\
\hline MSX LMC 1042 & 3.91 & -4.80 & 600 & 13 & 3 \\
\hline MSX LMC 1249 & 4.07 & -4.84 & 700 & 10 & 4 \\
\hline MSX LMC 50 & 3.95 & -4.51 & 650 & 33 & 2 \\
\hline MSX LMC 91 & 3.85 & -4.77 & 650 & 19 & 3 \\
\hline MSX LMC 93 & 3.88 & -4.82 & 700 & 17 & 3 \\
\hline MSX LMC 55 & 4.04 & -4.74 & 450 & 4 & 4 \\
\hline MSX LMC 45 & 4.31 & -4.30 & 650 & 28 & 1 \\
\hline MSX LMC 47 & 4.07 & -4.51 & 650 & 25 & 2 \\
\hline MSX LMC 219 & 4.03 & -4.58 & 700 & 25 & 2 \\
\hline MSX LMC 232 & 3.90 & -4.73 & 600 & 17 & 3 \\
\hline MSX LMC 223 & 4.00 & -4.54 & 700 & 30 & 2 \\
\hline MSX LMC 202 & 3.78 & -4.76 & 700 & 25 & 3 \\
\hline MSX LMC 225 & 4.01 & -4.48 & 650 & 32 & 2 \\
\hline MSX LMC 221 & 3.94 & -4.65 & 550 & 17 & 2 \\
\hline MSX LMC 349 & 3.90 & -4.54 & 700 & 37 & 2 \\
\hline MSX LMC 307 & 4.15 & -4.38 & 650 & 31 & 1 \\
\hline MSX LMC 341 & 3.80 & -4.75 & 700 & 25 & 3 \\
\hline MSX LMC 484 & 3.71 & -4.78 & 1000 & 31 & 3 \\
\hline MSX LMC 774 & 4.00 & -4.60 & 600 & 20 & 2 \\
\hline MSX LMC 677 & 3.88 & -4.85 & 700 & 15 & 3 \\
\hline MSX LMC 635 & 4.27 & -4.31 & 650 & 29 & 1 \\
\hline MSX LMC 692 & 4.01 & -4.70 & 700 & 18 & 4 \\
\hline MSX LMC 644 & 4.02 & -4.71 & 700 & 17 & 4 \\
\hline MSX LMC 654 & 3.88 & -4.67 & 700 & 20 & 2 \\
\hline MSX LMC 1780 & 4.07 & -4.35 & 600 & 37 & 1 \\
\hline MSX LMC 743 & 3.76 & -4.85 & 700 & 20 & 3 \\
\hline MSX LMC 872 & 3.94 & -4.61 & 700 & 28 & 2 \\
\hline MSX LMC 737 & 4.07 & -4.69 & 700 & 16 & 4 \\
\hline \multicolumn{6}{|c|}{ Cluster carbon stars from van Loon et al. (2005b) } \\
\hline NGC 1903-IR1 & 3.99 & -5.11 & 1300 & 9 & \\
\hline NGC 1903-IR2 & 3.84 & -5.43 & 1500 & 6 & \\
\hline NGC 1978-IR1 & 3.73 & -5.05 & 1000 & 9 & \\
\hline NGC 1978-IR2 & 3.77 & -4.80 & 1100 & 22 & \\
\hline \multicolumn{6}{|c|}{ LMC carbon-rich stars from Matsuura et al. (2005) } \\
\hline IRAS 04286-6937 & 3.95 & -4.43 & 420 & 15 & \\
\hline IRAS 04496-6958 & 4.61 & -4.54 & 720 & 6 & \\
\hline IRAS 04539-6821 & 3.94 & -4.54 & 700 & 27 & \\
\hline IRAS $04557-6753$ & 3.99 & -4.57 & 600 & 16 & \\
\hline IRAS 05112-6755 & 4.22 & -4.25 & 500 & 20 & \\
\hline BMB-R 46 & 4.19 & -5.27 & 700 & 1 & \\
\hline LI-LMC 1813 & 4.09 & -4.33 & 600 & 28 & \\
\hline
\end{tabular}

We also made a slight adjustment to the definition of the blue edge of the $3.1 \mu \mathrm{m}$ band.

The uncertainties in the equivalent width values are obtained from the standard deviations of the values for the eight oxygen-rich objects: $\sigma=170,2.23,3.03$ and $175 \AA$ in the equivalent widths at $3.1,3.57,3.70$ and $3.8 \mu \mathrm{m}$, respectively. This assumes that oxygen-rich objects have zero equivalent widths, which is not strictly true; the quoted uncertainties are therefore conservative estimates. The 3.57 and especially the

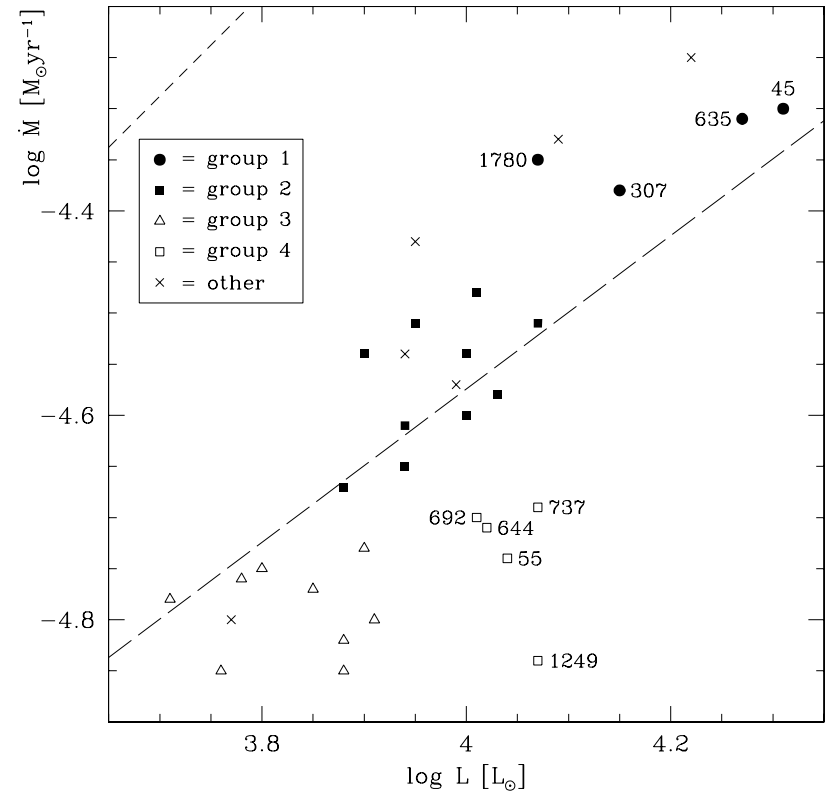

Fig. 7. Mass-loss rate versus luminosity diagram for IR carbon stars, which we use to subdivide the sample into four groups represented by different symbols. Stars from the cluster sample of van Loon et al. (2005b) and previously published spectra of Matsuura et al. (2005) are kept separate (crosses).

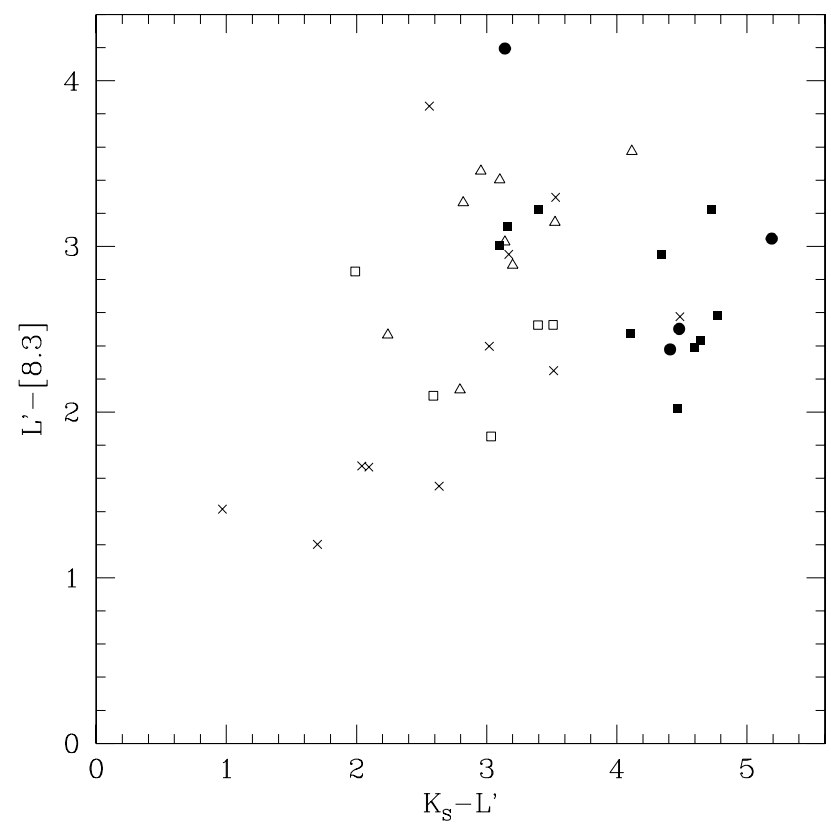

Fig. 8. $L^{\prime}-$ [8.3] versus $K_{\mathrm{s}}-L^{\prime}$ colour-colour diagram of the IR carbon stars. Redder colours are generally indicative of a larger optical depth of the circumstellar dust envelope, but there is significant scatter due to other (circum)stellar parameters and non-contemporaneous photometry. Symbols are as in Fig. 7.

$3.70 \mu \mathrm{m}$ features are quite difficult to measure in individual spectra.

All four groups of IR carbon stars show a very clear correlation between the strength of the $3.8 \mu \mathrm{m}$ band and that of the $3.1 \mu \mathrm{m}$ band (Fig. 12), with a Pearson correlation coefficient of $p=0.80$ : an increase in column density of $\mathrm{C}_{2} \mathrm{H}_{2}$ would make 


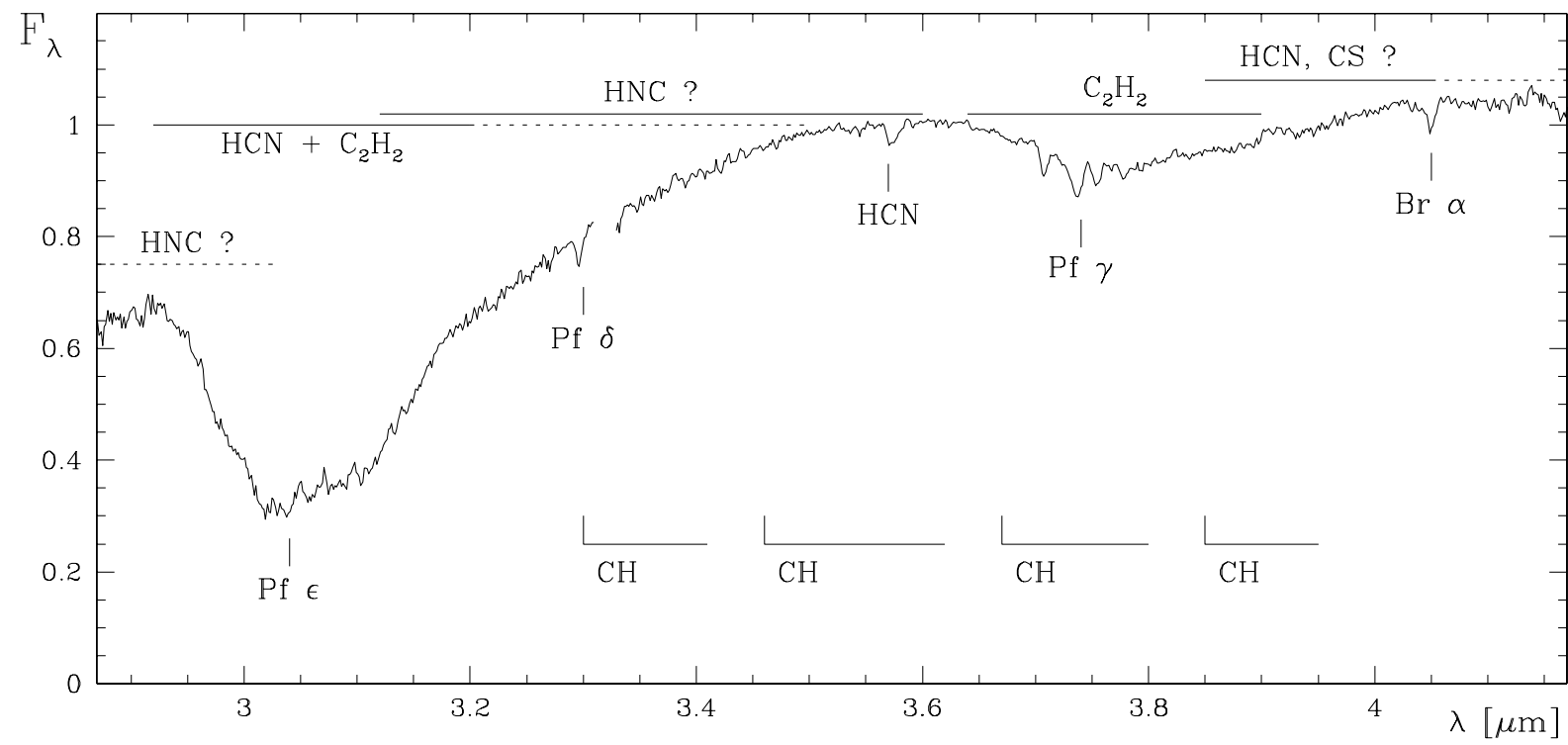

Fig. 9. Grand average of all IR carbon star spectra (Table 1), with identifications of the main features discussed in the text.

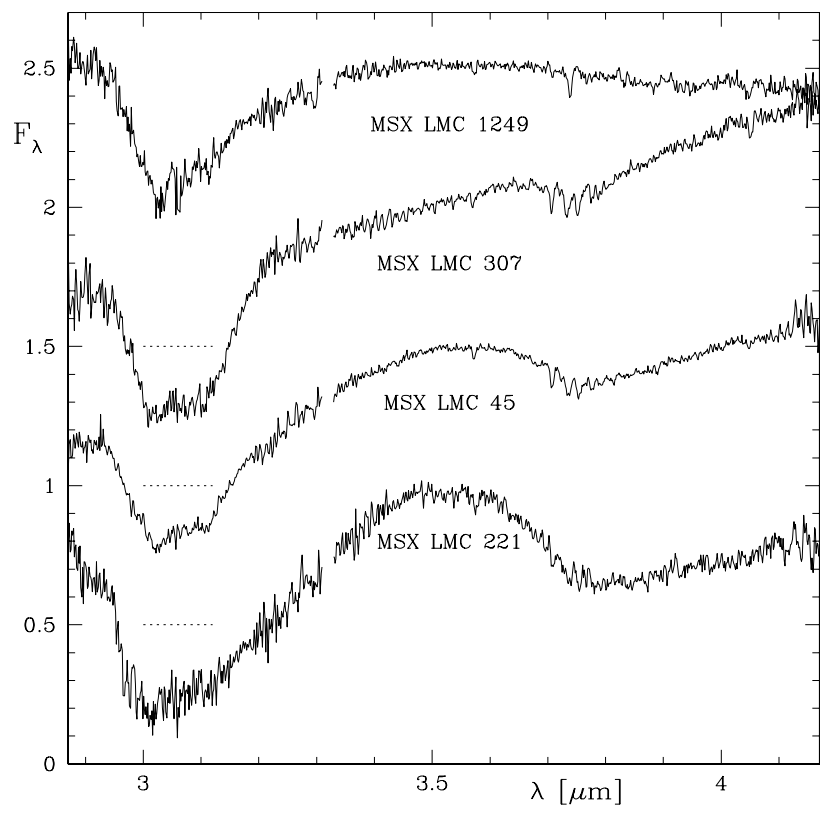

Fig. 10. Examples of four types of spectral appearance amongst the IR carbon stars.

both bands appear stronger. The $3.1 \mu \mathrm{m}$ band is always prominent, within a range in equivalent width of a factor two. The $3.8 \mu \mathrm{m}$ band is always seen in conjunction with strong $3.1 \mu \mathrm{m}$ absorption, $W_{3.1}>1000 \AA$ ( $p=0.85$, including the additional stars - crosses in Fig. 12), but it is not always visible in spectra with weaker $3.1 \mu \mathrm{m}$ absorption, when the $3.1 \mu \mathrm{m}$ band may be dominated by the contribution from $\mathrm{HCN}$.

For $W_{3.8}<450 \AA$, the correlation between the 3.70 and $3.8 \mu \mathrm{m}$ band strengths is much better $(p=0.41)$ than that between the 3.57 and $3.8 \mu \mathrm{m}$ band strengths $(p=0.11)$. This might imply that the $3.70 \mu \mathrm{m}$ band is associated with $\mathrm{C}_{2} \mathrm{H}_{2}$. At $W_{3.8}>450 \AA$ veiling and changes in excitation conditions (see below) may become important and affect the correlations.
Some of the scatter in the equivalent width distributions is intrinsic. For instance, MSX LMC 91 exhibits extremely strong 3.57 and $3.70 \mu \mathrm{m}$ bands but only moderately strong 3.1 and $3.8 \mu \mathrm{m}$ bands, whilst MSX LMC 221 shows exactly the opposite behaviour. Another extreme object is the relatively blue MSXLMC 1249, whose $3.1 \mu \mathrm{m}$ band resembles that of water ice (which is not expected to exist in a warm carbondominated chemistry) and which does not exhibit any 3.70 or $3.8 \mu \mathrm{m}$ absorption although it does show a small dip at $3.57 \mu \mathrm{m}$. The differences between extreme examples of 3-4 $\mu \mathrm{m}$ spectra (Fig. 10) are much greater than the subtle differences as a function of luminosity and mass-loss rate (Fig. 11), suggesting that other parameters influence the shape and strength of the molecular bands.

The shape of the spectrum between about 3.1 and $3.7 \mu \mathrm{m}$ can be dramatically different for different carbon stars (see Fig. 10), without it being clear what absorption band - if any - is responsible for this. To investigate this further we compute the spectral slopes, $s(\lambda)$, around $3.15,3.24,3.43$ and $3.57 \mu \mathrm{m}$ within the regions $3.12-3.18,3.20-3.28,3.36-3.50$ and 3.50-3.64 (excluding 3.56-3.58) $\mu \mathrm{m}$, respectively. We then compute the pair-wise differential spectral slopes, $s^{\prime}(\lambda)$ (Table 7), to quantify the degree to which the spectrum displays a "knee" such as is seen, for example, at $3.2 \mu \mathrm{m}$ in MSX LMC 307 or at $3.5 \mu \mathrm{m}$ in MSX LMC 221. If there is no change in spectral slope the differential spectral slope is zero.

The differential slopes at 3.2 and $3.5 \mu \mathrm{m}$ show a strong anticorrelation (Fig. 13; $p=-0.57$, or $p=-0.62$ if the noisier spectra of group 3 sources are omitted). There is a gradual transition between two extreme types of spectral shape: those with a narrow $3.1 \mu \mathrm{m}$ band and a flat continuum between 3.2 and $3.7 \mu \mathrm{m}$ ("cup"), and those with absorption extending from the $3.1 \mu \mathrm{m}$ band all the way until $3.5 \mu \mathrm{m}$ where it meets the continuum or dips into the $3.8 \mu \mathrm{m}$ band ("bowl"). Interestingly, the best examples of the "cup" type are all moderate to high massloss rate and luminosity objects (including IRAS 04286-6937) 


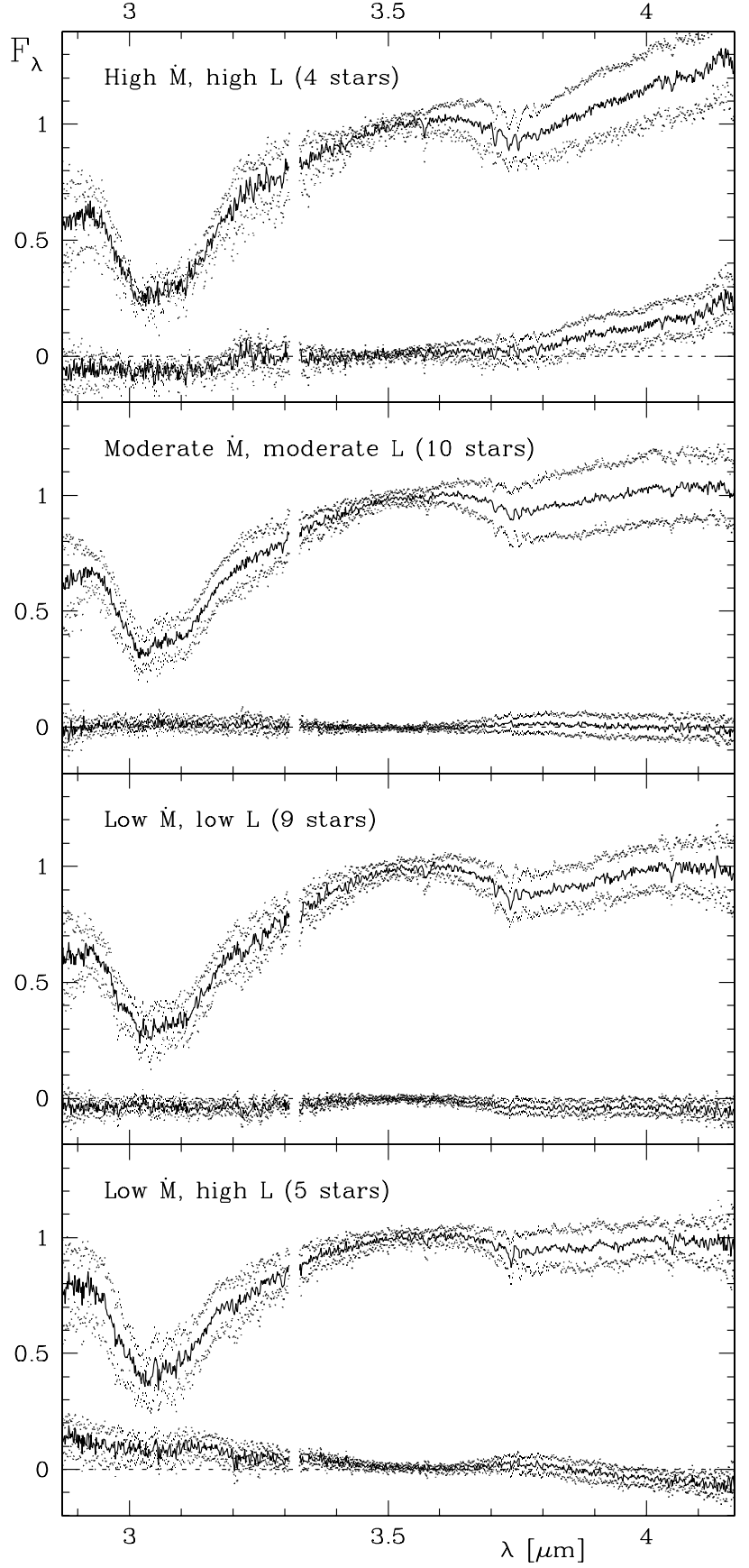

Fig. 11. Average spectra for the four groups of stars selected on the basis of their mass-loss rate and luminosity, plotted together with their difference spectra with respect to the grand average of all IR carbon star spectra (see Fig. 9). The dots indicate the dispersion in the group averages and the error in the mean difference between the group and grand averages.

although the "bowl" type also occurs amongst these classes of stars.

The strength of the $3.70 \mu \mathrm{m}$ feature is correlated with the differential slope at $3.2 \mu \mathrm{m}$ (Fig. 14; $p=0.45$ ). This suggests that whatever causes the broad absorption between 3.2 and $3.5 \mu \mathrm{m}$ is anti-correlated with the $3.70 \mu \mathrm{m}$ feature. On the other hand the strength of the $3.8 \mu \mathrm{m}$ band is correlated with the differential slope at $3.5 \mu \mathrm{m}(p=0.72)$ and mildly anti-correlated
Table 5. The band and blue/red continuum definitions for the computation of equivalent widths. Wavelengths are in $\mu \mathrm{m}$.

\begin{tabular}{lccc}
\hline \hline Feature & band & blue continuum & red continuum \\
\hline 3.1 & $2.950-3.350$ & $2.900-2.950$ & $3.350-3.400$ \\
3.57 & $3.560-3.580$ & $3.510-3.560$ & $3.580-3.630$ \\
3.70 & $3.700-3.715$ & $3.680-3.700$ & $3.715-3.720$ \\
3.8 & $3.600-4.000$ & $3.500-3.600$ & $4.000-4.100$ \\
\hline
\end{tabular}

with that at $3.2 \mu \mathrm{m}(p=-0.33)$, suggesting a link between the 3.2-3.5 and $3.8 \mu \mathrm{m}$ absorptions. We interpret this as an effect of different excitation conditions. A bowl-shaped $3.1 \mu \mathrm{m}$ band indicates a high excitation temperature, which would then also result in broad absorption by $\mathrm{C}_{2} \mathrm{H}_{2}$ at $3.8 \mu \mathrm{m}$. A low excitation temperature would result in narrow absorption features of $\mathrm{C}_{2} \mathrm{H}_{2}$, between 3.70-3.78 $\mu \mathrm{m}$.

\subsection{Relation between the molecular atmosphere and circumstellar dust envelope}

Can some of the differences in the molecular bands be reflected in differences in the circumstellar dust envelope? Stars with the highest mass-loss rates and luminosities always have strong $3.8 \mu \mathrm{m}$ bands and almost always do they also have a conspicuous $3.70 \mu \mathrm{m}$ feature (Fig. 15). Stars with low mass-loss rates of $\dot{M}<10^{-5} M_{\odot} \mathrm{yr}^{-1}$ tend to have weak or absent 3.70 and $3.8 \mu \mathrm{m}$ absorptions whilst the $3.57 \mu \mathrm{m}$ band is often visible in these stars.

At high mass-loss rates of $\dot{M}>2.5 \times 10^{-5} M_{\odot} \mathrm{yr}^{-1}$ the 3.1, 3.57 and $3.8 \mu \mathrm{m}$ absorption bands increase in strength with increasing mass-loss rate. However, at lower mass-loss rates the bands are generally stronger than around $3 \times 10^{-5} M_{\odot} \mathrm{yr}^{-1}$, albeit with considerable spread. The same trends are seen for the $3.70 \mu \mathrm{m}$ band. This probably reflects a competition between the effect of veiling (reducing the equivalent width) and an optically thicker atmosphere in connection with a higher mass-loss rate (increasing the equivalent width).

Dust column densities increase with increasing mass-loss rate but also with decreasing luminosity as low-luminosity objects are more compact. Molecular column densities may be affected in a similar way, and the dust optical depth might therefore show a stronger correlation with the molecular column density than either the mass-loss rate or luminosity would do. The 3.1 and $3.8 \mu \mathrm{m}$ bands become weaker at progressively larger dust optical depth (Fig. 16). This is likely due to veiling by the increasing contribution of dust emission. The decrease in equivalent width is indeed larger at $3.8 \mu \mathrm{m}$ than at $3.1 \mu \mathrm{m}$. The 3.57 and $3.70 \mu \mathrm{m}$ equivalent width seems to remain constant, though, which may suggest that they would be seen to increase with optical depth if the effects of veiling were to be removed. At relatively modest optical depth, $\tau_{\mathrm{V}}<10$, the $3.57 \mu \mathrm{m}$ band is often quite strong but the $3.70 \mu \mathrm{m}$ band is often absent.

The effects of veiling by dust emission can in principle be removed, as DUSTY returns the fractional contribution of the dust emission to the total flux density as a function of wavelength. We tabulate these values at wavelengths of 3.0, 3.5 and $4.0 \mu \mathrm{m}$ (Table 7). Unfortunately, these fractions are 
Table 6. Equivalent widths (in $\AA$ ). The chemical classification into carbon stars (C) and oxygen-rich stars (o) is based on the presence or absence of the $3.1 \mu \mathrm{m}$ feature, supplemented by information from the literature.

\begin{tabular}{|c|c|c|c|c|c|}
\hline Name & $W_{3.1}$ & $W_{3.57}$ & $W_{3.70}$ & $W_{3.8}$ & $\mathrm{C} / \mathrm{o}$ \\
\hline \multicolumn{6}{|l|}{ IR carbon stars } \\
\hline MSX LMC 1042 & 1333 & -0.23 & 5.12 & 405 & $\mathrm{C}$ \\
\hline MSX LMC 1249 & 889 & 1.79 & 0.04 & 34 & $\mathrm{C}$ \\
\hline MSX LMC 50 & 786 & 5.04 & 2.16 & 14 & $\mathrm{C}$ \\
\hline MSX LMC 91 & 1189 & 8.97 & 7.10 & 208 & $\mathrm{C}$ \\
\hline MSX LMC 93 & 821 & 2.00 & 1.58 & -14 & $\mathrm{C}$ \\
\hline MSX LMC 55 & 1078 & 6.73 & 2.45 & 221 & $\mathrm{C}$ \\
\hline MSX LMC 45 & 1236 & 1.72 & 4.79 & 322 & $\mathrm{C}$ \\
\hline MSX LMC 47 & 1103 & 0.46 & 2.87 & 144 & $\mathrm{C}$ \\
\hline MSX LMC 219 & 869 & 0.44 & 1.25 & 133 & $\mathrm{C}$ \\
\hline MSX LMC 232 & 1302 & 0.29 & 1.21 & 182 & $\mathrm{C}$ \\
\hline MSX LMC 223 & 1016 & 0.96 & 2.76 & 36 & $\mathrm{C}$ \\
\hline MSX LMC 202 & 1371 & 8.05 & 0.11 & 472 & $\mathrm{C}$ \\
\hline MSX LMC 225 & 1023 & 0.37 & 2.87 & 101 & $\mathrm{C}$ \\
\hline MSX LMC 221 & 1783 & 4.38 & 3.11 & 588 & $\mathrm{C}$ \\
\hline MSX LMC 349 & 877 & -0.62 & 0.02 & 217 & $\mathrm{C}$ \\
\hline MSX LMC 307 & 1165 & 2.96 & 5.03 & 165 & $\mathrm{C}$ \\
\hline MSX LMC 341 & 949 & 4.52 & 6.75 & 191 & $\mathrm{C}$ \\
\hline MSX LMC 484 & 1208 & 6.51 & 3.76 & 140 & $\mathrm{C}$ \\
\hline MSX LMC 774 & 1144 & 2.80 & 5.03 & 185 & $\mathrm{C}$ \\
\hline MSX LMC 677 & 1384 & 3.07 & 0.52 & 182 & $\mathrm{C}$ \\
\hline MSX LMC 635 & 1267 & 7.49 & 3.29 & 400 & $\mathrm{C}$ \\
\hline MSX LMC 692 & 1023 & 2.44 & 1.40 & -22 & $\mathrm{C}$ \\
\hline MSX LMC 644 & 1005 & 1.75 & 2.91 & 40 & $\mathrm{C}$ \\
\hline MSX LMC 654 & 1336 & 8.63 & 6.09 & 157 & $\mathrm{C}$ \\
\hline MSX LMC 1780 & 1227 & 3.36 & 6.15 & 258 & $\mathrm{C}$ \\
\hline MSX LMC 743 & 1485 & 0.73 & 3.54 & 377 & $\mathrm{C}$ \\
\hline MSX LMC 872 & 1046 & 1.55 & 2.35 & 21 & $\mathrm{C}$ \\
\hline MSX LMC 737 & 1346 & 4.28 & 3.07 & 262 & $\mathrm{C}$ \\
\hline \multicolumn{6}{|c|}{ IR objects of unknown type } \\
\hline MSX LMC 196 & 493 & 5.20 & 2.35 & -67 & C? \\
\hline MSX LMC 733 & 375 & 4.50 & -1.04 & -142 & $\mathrm{o} ?$ \\
\hline \multicolumn{6}{|c|}{ Cluster objects from van Loon et al. (2005b) } \\
\hline NGC 1903-IR1 & 1537 & 6.74 & 5.18 & 448 & $\mathrm{C}$ \\
\hline NGC 1903-IR2 & 870 & 3.87 & 1.16 & 70 & $\mathrm{C}$ \\
\hline NGC 1984-IR1 & 116 & 0.30 & -0.69 & -29 & o \\
\hline NGC 1984-IR2 & 5 & -0.60 & -0.89 & 248 & o \\
\hline NGC 1978-IR1 & 1110 & 0.70 & -1.89 & 41 & $\mathrm{C}$ \\
\hline NGC 1978-IR2 & 1349 & 3.20 & 5.19 & 267 & $\mathrm{C}$ \\
\hline \multicolumn{6}{|c|}{ Serendipitous objects } \\
\hline MSX LMC 50 B & 99 & 0.94 & 5.25 & 37 & o \\
\hline HV 11977 & 136 & -2.04 & 6.56 & 72 & o \\
\hline MSX LMC 349 B & 1485 & 3.50 & -1.57 & -23 & $\mathrm{C}$ \\
\hline HV 2532 & -38 & 1.07 & 1.08 & 55 & o \\
\hline MSX LMC $1780 \mathrm{~B}$ & 220 & -0.75 & -1.80 & 198 & o \\
\hline \multicolumn{6}{|c|}{ IR objects from Matsuura et al. (2005) } \\
\hline IRAS 04286-6937 & 508 & -3.66 & 7.14 & 330 & $\mathrm{C}$ \\
\hline IRAS 04496-6958 & 1726 & 7.77 & -1.87 & 735 & $\mathrm{C}$ \\
\hline IRAS 04539-6821 & 771 & 1.89 & 2.57 & 183 & $\mathrm{C}$ \\
\hline IRAS 04557-6753 & 1309 & 0.65 & 3.96 & 542 & $\mathrm{C}$ \\
\hline IRAS 05112-6755 & 887 & 0.90 & 5.28 & 224 & $\mathrm{C}$ \\
\hline IRAS 05128-6455 & -364 & 1.98 & -1.36 & -228 & o \\
\hline IRAS 05148-6730 & -95 & -5.61 & -1.23 & 286 & $\mathrm{o}$ \\
\hline BMB-R 46 & 1161 & 12.93 & -10.33 & 84 & $\mathrm{C}$ \\
\hline LI-LMC 1813 & 1273 & 16.55 & -4.39 & 151 & $\mathrm{C}$ \\
\hline
\end{tabular}

ill-determined: they depend sensitively on the amount and temperature of the warmest dust, which is also the most timevariable component. Hence correcting the equivalent widths in this way increases the overall scatter in a sub-set of the sample of carbon stars (Fig. 17), which is especially due to stars with fairly modest mass-loss rates. However, for at least half the sample of stars the scatter is reduced and the $3.1 \mu \mathrm{m}$ equivalent widths of the four groups of stars are more in line with each other (Fig. 17). This suggests that veiling is the main driver behind variations of the $3.1 \mu \mathrm{m}$ equivalent width with mass-loss rate and luminosity.

Consequently the photospheric $3.1 \mu \mathrm{m}$ equivalent width seems to depend very little on stellar or circumstellar parameters, and we propose that this band may be saturated for the MSX-selected sample of IR carbon stars. Comparing the dust continuum contribution (Table 7) with the maximum depth of the $3.1 \mu \mathrm{m}$ absorption in the spectra of Fig. 2, it appears that part of the absorption may in fact be seen against the dust continuum. If true this would imply a dense molecular atmosphere with a scale height of order a few stellar radii, which is seen in absorption against the dust emission arising from behind and adjacent to the limb of the star. The same may be true for the $3.8 \mu \mathrm{m}$ band in some stars.

A large molecular zone is also implied by the low temperature at the dust formation radius that was required to fit the SEDs. The molecular atmosphere must extend to at least the dust formation radius for dust to form, and a lower temperature at that radius implies that the dust forms farther from the star. If the temperature had been underestimated, then the veiling of the absorption bands by the dust continuum around 3-4 $\mu \mathrm{m}$ would also have been underestimated. This would make the case even more convincing for part of the molecular absorption to be seen against background dust continuum emission.

A weak trend can be seen in the differential spectral slope at $3.5 \mu \mathrm{m}$ as a function of mass-loss rate (Fig. 18), suggesting that stars enduring higher mass-loss rates have a "cup"shaped 3.2-3.5 $\mu \mathrm{m}$ spectrum. At high mass-loss rate the $3.1 \mu \mathrm{m}$ band may on average be formed higher above the stellar photosphere, at a lower excitation temperature, which would explain the narrower shape ("cup"). On the other hand, the two MSX-selected stars with the highest mass-loss rates defy such relationship.

\subsection{Pulsation and excitation conditions}

Pulsation is known to affect the absorption bands. The difficulty with investigating dependencies on pulsational properties is that the effects have a highly time-variable component. But the pulsation may also affect time-averaged properties, that we can study here. In addition to the stars with known pulsation properties listed in Table 2, pulsation periods and amplitudes are known for IRAS 04496-6958 and IRAS 04557-6753 (Whitelock et al. 2003) and rough estimates for LI-LMC 1813 (van Loon et al. 2003). The $L^{\prime}$-band amplitude of $1.6 \mathrm{mag}$ for LI-LMC 1813 is scaled to a $K_{\mathrm{s}}$-band amplitude of $\Delta K_{\mathrm{s}} \sim$ 2.0 mag (cf. Whitelock et al. 2003). 

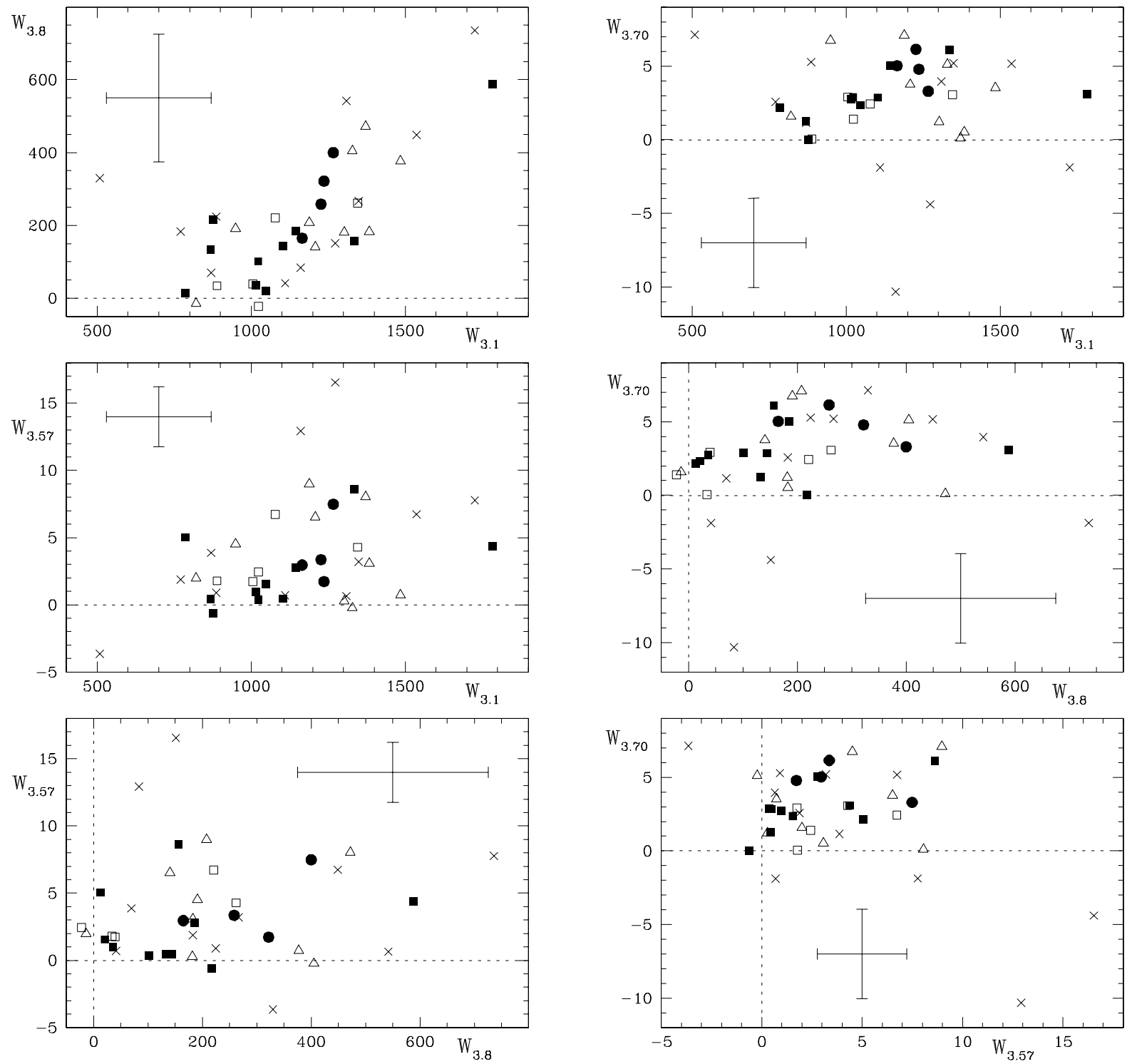

Fig. 12. Equivalent widths of the $3.1,3.57,3.70$ and $3.8 \mu \mathrm{m}$ bands (in $\AA$ ) plotted versus each other. A typical errorbar is plotted in each panel. The strongest correlation is between the 3.1 and $3.8 \mu \mathrm{m}$ bands (top left). Symbols are as in Fig. 7 .

The equivalent width (at 3.1 and $3.8 \mu \mathrm{m}$ ) shows no correlation with the photometric amplitude, $\Delta K_{\mathrm{s}}$ (Fig. 19, left panel; $p=-0.15$ and $p=-0.21$, respectively). However, the photometric amplitude is relative to the star's luminosity. An absolute quantity that more directly measures the amount of energy involved in the pulsation is the energy injection rate (van Loon 2002):

$\dot{E}=\frac{L}{2} \times\left\{\frac{\exp \left(\Delta K_{\mathrm{s}} / 2.5\right)-1}{\exp \left(\Delta K_{\mathrm{s}} / 2.5\right)+1}\right\}$

This is the fraction of the bolometric luminosity that is used in expanding the star, assuming that the amplitude at $K_{\mathrm{s}}$ measures the bolometric amplitude and that the luminosity varies sinusoidally. It is by definition limited to $0<\dot{E}<L / 2$. The equivalent widths at 3.1 and $3.8 \mu \mathrm{m}$ show a weak correlation with $\dot{E}$ (Fig. 19, right panel; $p=0.50$ and $p=0.41$, respectively), suggesting a positive link between stronger pulsation and a larger column density of the molecular atmosphere - in particular of $\mathrm{C}_{2} \mathrm{H}_{2}$.

Pulsation also affects the excitation conditions. The fact that the $3.1 \mu \mathrm{m}$ band is narrower at high mass-loss rates is because both are related to the increased scale height as a consequence of strong pulsation. Indeed (Fig. 20), the differential spectral slope at $3.2 \mu \mathrm{m}$ is weakly correlated with the $K_{\mathrm{s}}$-band amplitude $(p=0.26)$ and pulsation period $(p=0.53)$ whilst the differential spectral slope at $3.5 \mu \mathrm{m}$ shows the opposite behaviour ( $p=-0.61$ and $p=-0.45$, respectively). This suggests that the $3.1 \mu \mathrm{m}$ absorption profile is narrower at larger photometric amplitude and longer pulsation period, supporting evidence for a lower excitation temperature in a more extended molecular atmosphere. There is a possible competing mechanism associated with the pulsation: shocks induced by strong pulsation cause the band to broaden again (Yamamura et al. 1997). This might explain the "bowl"-shaped absorption in the two MSX-selected stars with the highest mass-loss rates. 
Table 7. Differential spectral slopes $s^{\prime}(3.2)=s(3.15)-s(3.24)$ and $s^{\prime}(3.5)=s(3.43)-s(3.57)$ (see text), and the fractional contributions of the dust emission, for carbon stars.

\begin{tabular}{lccccc}
\hline \hline Name & $s^{\prime}(3.2)$ & $s^{\prime}(3.5)$ & $f_{3.0}$ & $f_{3.5}$ & $f_{4.0}$ \\
\hline IR carbon stars & & & & & \\
MSX LMC 1042 & 2.59 & 0.61 & 0.36 & 0.56 & 0.70 \\
MSX LMC 1249 & 1.65 & 0.28 & 0.48 & 0.64 & 0.74 \\
MSX LMC 50 & 2.42 & 0.38 & 0.47 & 0.67 & 0.78 \\
MSX LMC 91 & 1.73 & 1.01 & 0.45 & 0.64 & 0.76 \\
MSX LMC 93 & 1.73 & 0.44 & 0.52 & 0.68 & 0.78 \\
MSX LMC 55 & 2.32 & 0.57 & 0.80 & 0.86 & 0.89 \\
MSX LMC 45 & 1.50 & 0.96 & 0.47 & 0.66 & 0.78 \\
MSX LMC 47 & 1.68 & 0.28 & 0.46 & 0.65 & 0.77 \\
MSX LMC 219 & 1.29 & 0.82 & 0.54 & 0.70 & 0.80 \\
MSX LMC 232 & 1.77 & 0.24 & 0.37 & 0.57 & 0.71 \\
MSX LMC 223 & 3.78 & 0.31 & 0.55 & 0.71 & 0.81 \\
MSX LMC 202 & 0.23 & 1.71 & 0.54 & 0.70 & 0.80 \\
MSX LMC 225 & 1.88 & 0.36 & 0.47 & 0.67 & 0.78 \\
MSX LMC 221 & 1.10 & 1.49 & 0.37 & 0.57 & 0.71 \\
MSX LMC 349 & 1.40 & 0.66 & 0.56 & 0.73 & 0.82 \\
MSX LMC 307 & 5.14 & 0.07 & 0.47 & 0.66 & 0.78 \\
MSX LMC 341 & 2.68 & 1.06 & 0.54 & 0.70 & 0.80 \\
MSX LMC 484 & 2.16 & 0.83 & 0.85 & 0.89 & 0.92 \\
MSX LMC 774 & 2.26 & 0.64 & 0.37 & 0.58 & 0.72 \\
MSX LMC 677 & 2.72 & 1.05 & 0.51 & 0.67 & 0.78 \\
MSX LMC 635 & 1.03 & 1.60 & 0.47 & 0.66 & 0.78 \\
MSX LMC 692 & 1.84 & 0.53 & 0.52 & 0.68 & 0.78 \\
MSX LMC 644 & 3.01 & 0.38 & 0.52 & 0.68 & 0.78 \\
MSX LMC 654 & 4.50 & 0.46 & 0.57 & 0.73 & 0.82 \\
MSX LMC 1780 & 3.30 & 0.21 & 0.38 & 0.60 & 0.75 \\
MSX LMC 743 & 1.55 & 1.23 & 0.53 & 0.69 & 0.79 \\
MSX LMC 872 & 1.36 & 0.53 & 0.54 & 0.71 & 0.81 \\
MSX LMC 737 & 0.47 & 0.82 & 0.51 & 0.67 & 0.77 \\
\hline Cluster carbon stars from van Loon et al. $(2005 b)$ & \\
NGC 1903-IR1 & 0.60 & 1.04 & 0.79 & 0.83 & 0.85 \\
NGC 1903-IR2 & -1.76 & 0.53 & 0.74 & 0.77 & 0.79 \\
NGC 1978-IR1 & 2.09 & 0.78 & 0.63 & 0.71 & 0.76 \\
NGC 1978-IR2 & 2.98 & 0.97 & 0.76 & 0.82 & 0.86 \\
\hline IR carbon-rich stars from Matsuura et al. $(2005)$ & & \\
IRAS 04286-6937 & 5.18 & 0.10 & 0.06 & 0.19 & 0.38 \\
IRAS 04496-6958 & 1.57 & 1.08 & 0.48 & 0.62 & 0.72 \\
IRAS 04539-6821 & 2.25 & 0.05 & 0.51 & 0.68 & 0.78 \\
IRAS 04557-6753 & 3.43 & 0.76 & 0.44 & 0.65 & 0.78 \\
IRAS 05112-6755 & 1.38 & 0.18 & 0.18 & 0.38 & 0.57 \\
BMB-R 46 & 0.06 & 1.20 & 0.16 & 0.25 & 0.34 \\
& 2.06 & 0.72 & 0.38 & 0.59 & 0.74 \\
\hline
\end{tabular}

\section{Discussion}

\subsection{The population of dust-enshrouded carbon stars}

The population of visually bright carbon stars in the LMC, selected on the basis of objective prism surveys, is estimated to be $N_{\text {vis }} \simeq 9000$ (Kontizas et al. 2001). In the galaxy, many carbon stars become extremely obscured by the very opaque carbon dust they produce when they enter the superwind stage with $\dot{M}>10^{-5} M_{\odot} \mathrm{yr}^{-1}$ (e.g., Jura \& Kleinmann 1990). These objects would escape detection in the optical and (shallow,

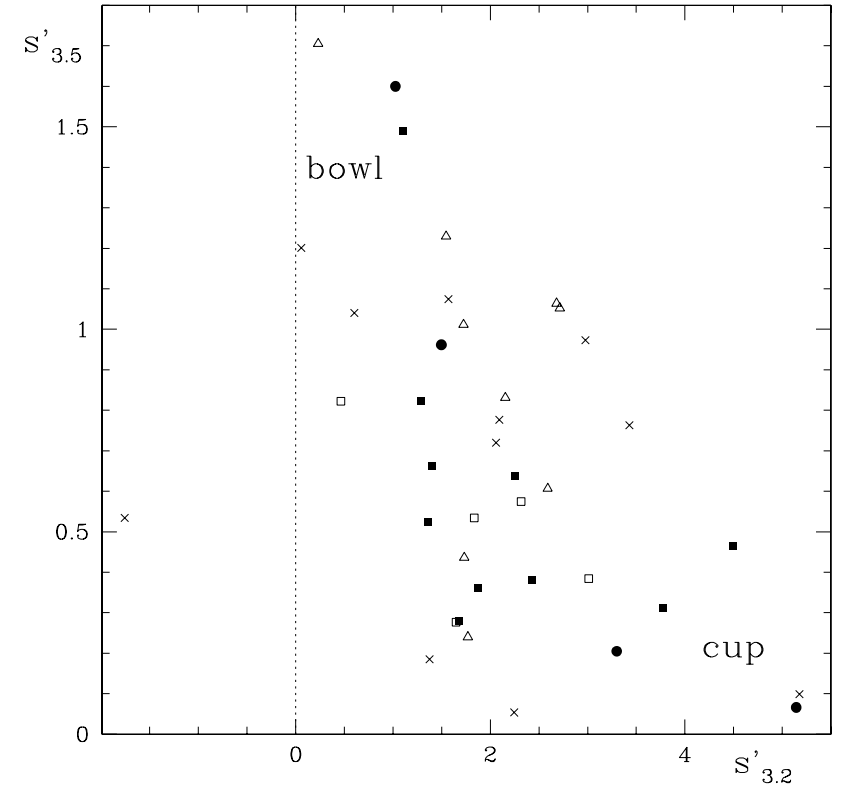

Fig. 13. Change in slope around $3.5 \mu \mathrm{m}$ versus that around $3.2 \mu \mathrm{m}$; there is a clear correlation. Symbols are as in Fig. 7.

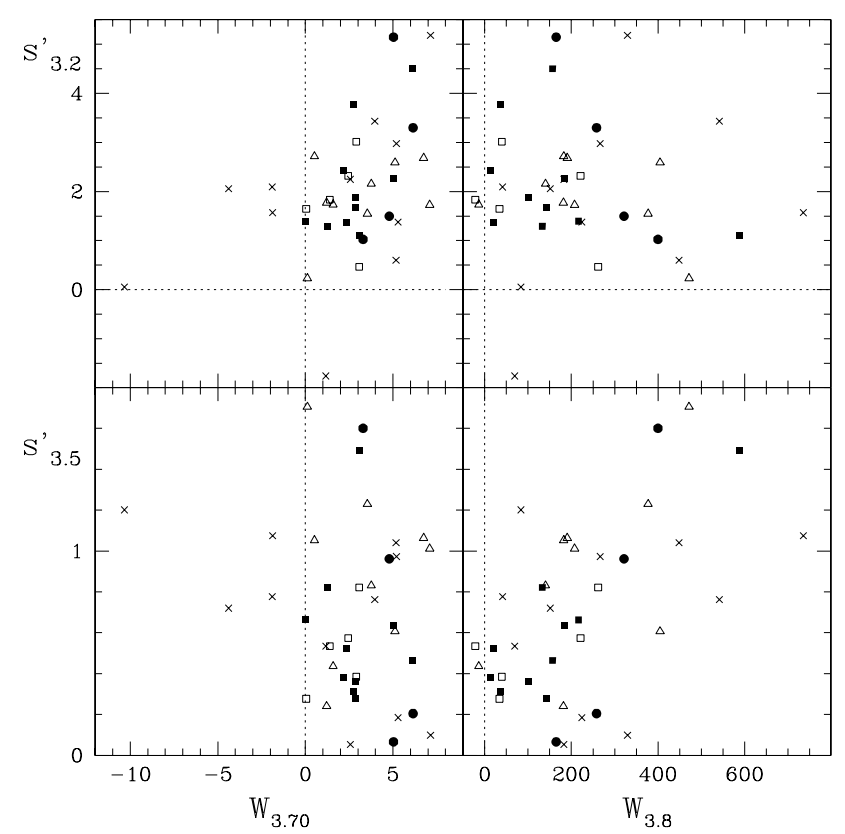

Fig. 14. Change in slope around 3.2 and $3.5 \mu \mathrm{m}$ versus the equivalent width of the 3.70 and $3.8 \mu \mathrm{m}$ bands. The $3.70 \mu \mathrm{m}$ band is generally stronger for larger $s_{3.2}^{\prime}$ and smaller $s_{3.5}^{\prime}$, whilst the $3.80 \mu \mathrm{m}$ band shows the opposite behaviour. Symbols are as in Fig. 7.

wide-field) near-IR surveys in the LMC but could stand out at mid-IR wavelengths where their dust envelopes shine brightly. The IRAS Point Source Catalogue was searched for obscured AGB stars in the LMC, and although several hundred such candidates were found (Loup et al. 1997) only a few dozen were successfully identified with carbon stars, with another few dozen oxygen-rich massive AGB stars or red supergiants (Reid et al. 1990; Wood et al. 1992; Zijlstra et al. 1996; van Loon et al. 1997). This was but a small fraction of the expected population of superwind AGB stars. However, the relatively deep 


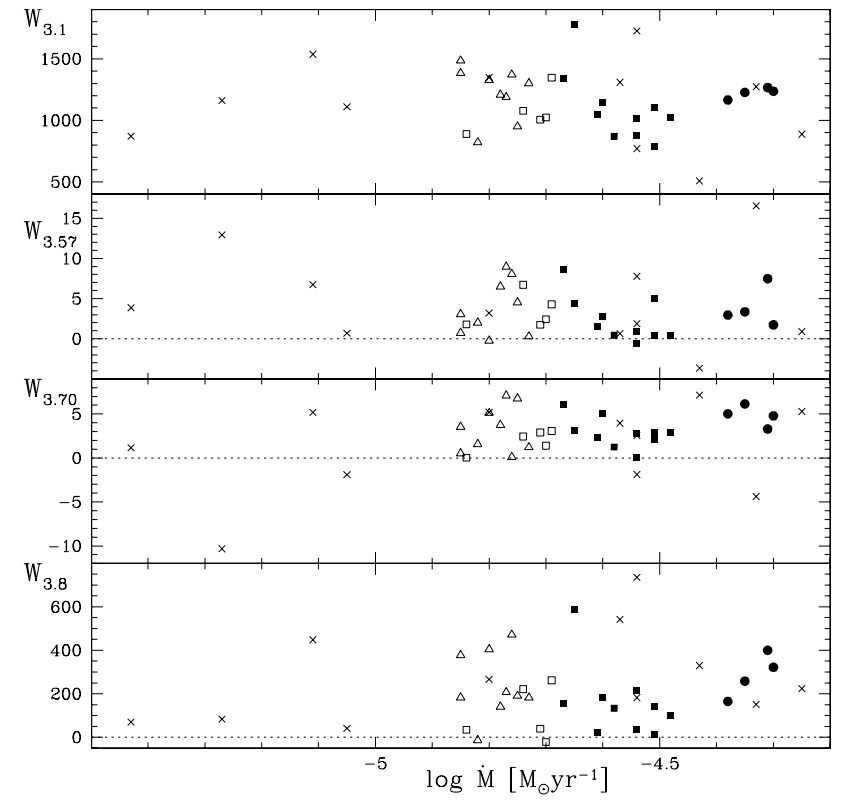

Fig. 15. Equivalent widths versus mass-loss rate. There is no clear correlation other than that the absorption is generally strong at the highest mass-loss rates. Symbols are as in Fig. 7.

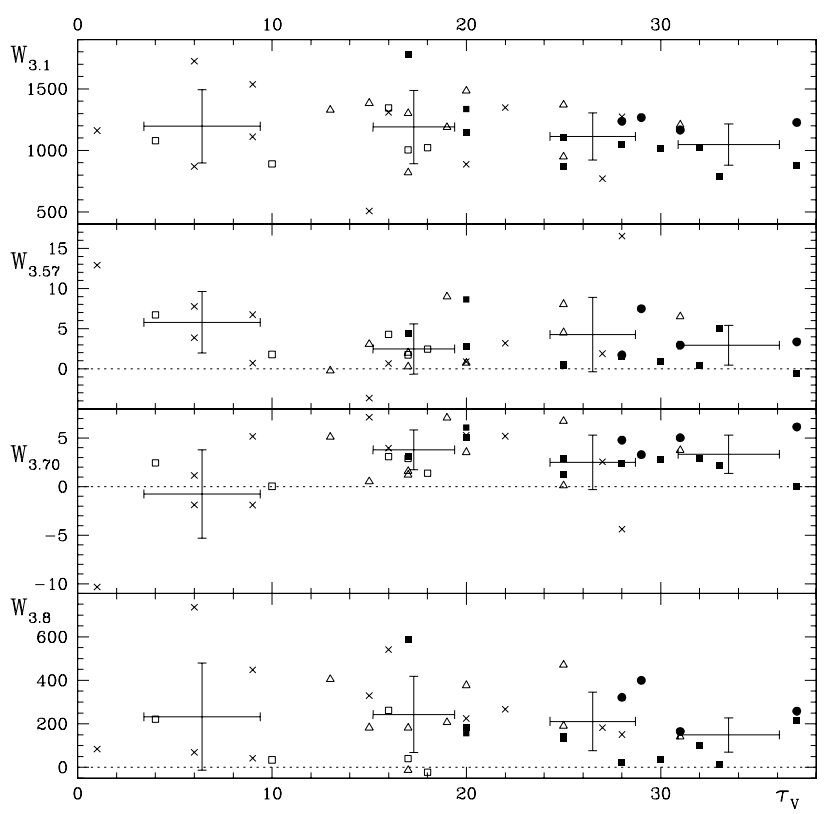

Fig. 16. Equivalent widths versus dust optical depth. The error bars refer to the mean values and standard deviations in the intervals $\tau_{\mathrm{V}} \in$ $\langle 0,10],\langle 10,20],\langle 20,30]$ and $\langle 30, \rightarrow\rangle$. There are weak trends in the 3.1 and $3.8 \mu \mathrm{m}$ bands to diminish in strength with greater optical depth. Symbols are as in Fig. 7.

near-IR imaging of IRAS positions by van Loon et al. (1997) recovered counterparts all the way down to their detection limit of $K_{\mathrm{s}}<18 \mathrm{mag}$, whilst leaving half of their IRAS sample unidentified. Most of the faint counterparts had colours of carbon stars - later spectroscopic data confirmed their carbon star nature (van Loon et al. 1999a; Trams et al. 1999). Van Loon et al. (1997) took this as evidence for a hitherto uncovered population of very obscured carbon stars.

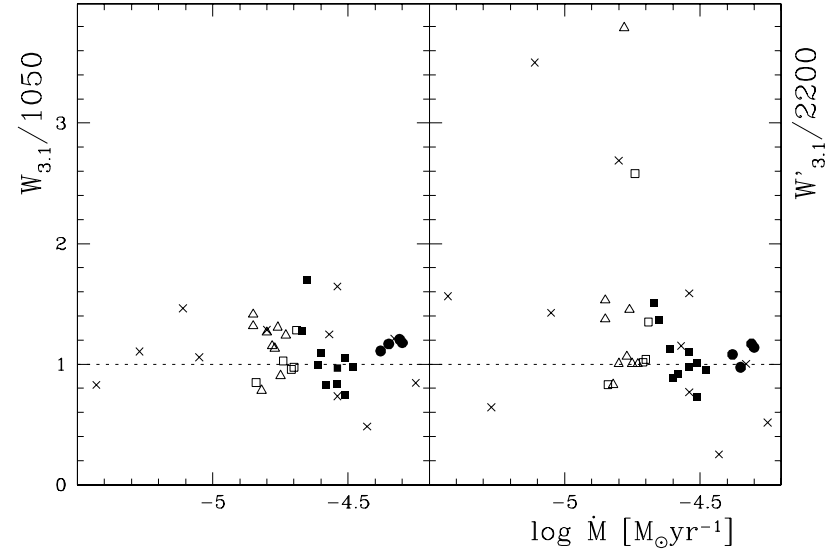

Fig. 17. Equivalent width of the $3.1 \mu \mathrm{m}$ band versus mass-loss rate before (left) and after (right) correction for dust emission, normalised to the peak in the distribution. Removing the dust emission creates a flatter and tighter distribution around unity for about half of the sample, but it creates large scatter for the remainder - many of which have low mass-loss rates. Symbols are as in Fig. 7.

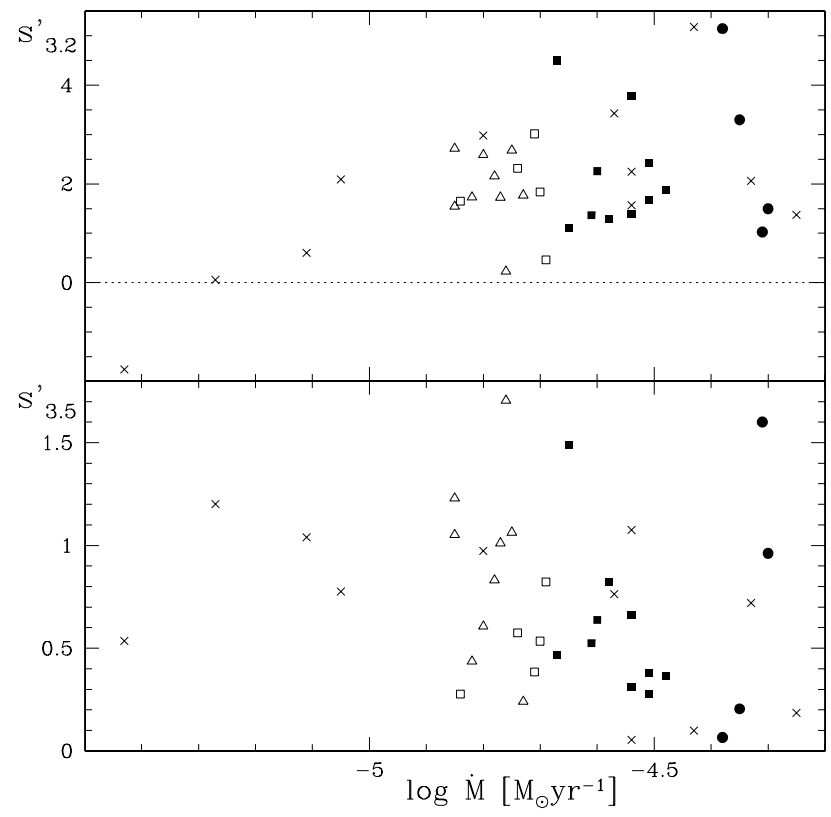

Fig. 18. Change in slope around 3.2 and $3.5 \mu \mathrm{m}$ versus mass-loss rate. There is a trend for the spectral shape in the $3.1-3.7 \mu \mathrm{m}$ region to turn from "bowl" to "cup", although the two MSX-selected carbon stars with the highest mass-loss rates exhibit a "bowl" shaped spectrum. Symbols are as in Fig. 7.

The MSX Point Source Catalogue at $8.3 \mu \mathrm{m}$ was crossidentified with the $2 \mathrm{MASS} J H K_{\mathrm{s}}$ catalogue to reveal the largest selection to date of candidate superwind AGB stars in the LMC (Egan et al. 2001; Wood \& Cohen 2001). Several hundred were found, with more than a hundred remaining without near-IR counterpart. Given the result of van Loon et al. (1997) and the shallower depth of 2MASS at $K_{\mathrm{s}}<15$ mag we can expect many of the MSX-selected candidate AGB stars and unidentified stellar MSX sources to be dust-enshrouded (IR) carbon stars. We here provide supporting evidence for this hypothesis, as our selection of targets from the MSX-2MASS compilation 


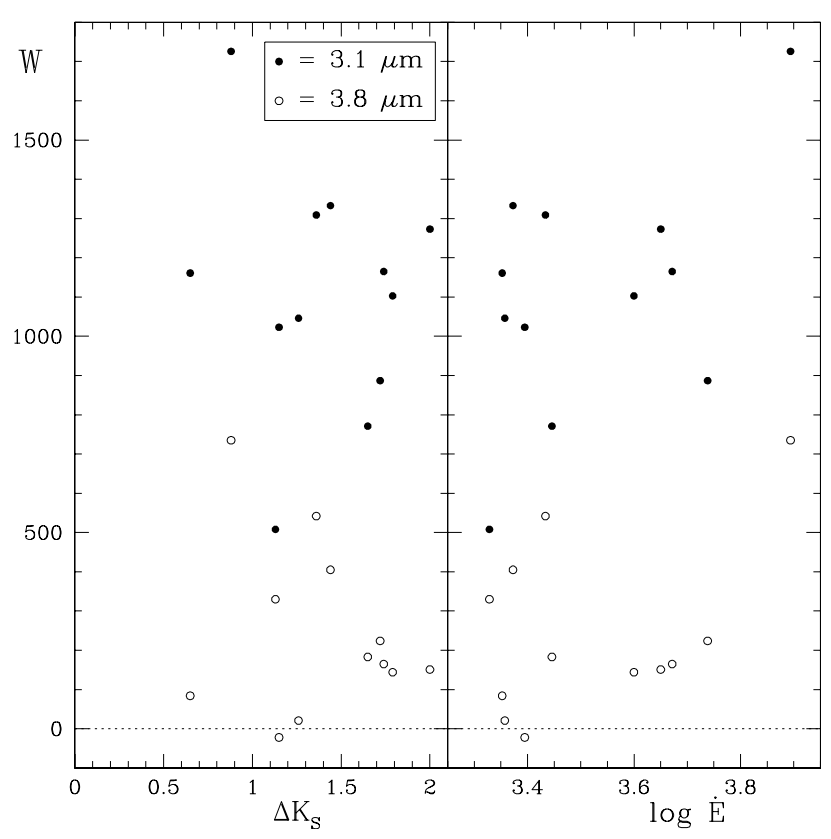

Fig. 19. Equivalent widths at $3.1 \mu \mathrm{m}$ (solid symbols) and $3.8 \mu \mathrm{m}$ (open symbols) versus $K_{\mathrm{s}}$-band pulsation amplitude (left panel), and versus pulsational energy injection rate per cycle, $\dot{E}$ (right panel), in logarithmic units of $L_{\odot}$. Whilst no correlation is seen with amplitude, the equivalent width is larger for larger energy injection rates.

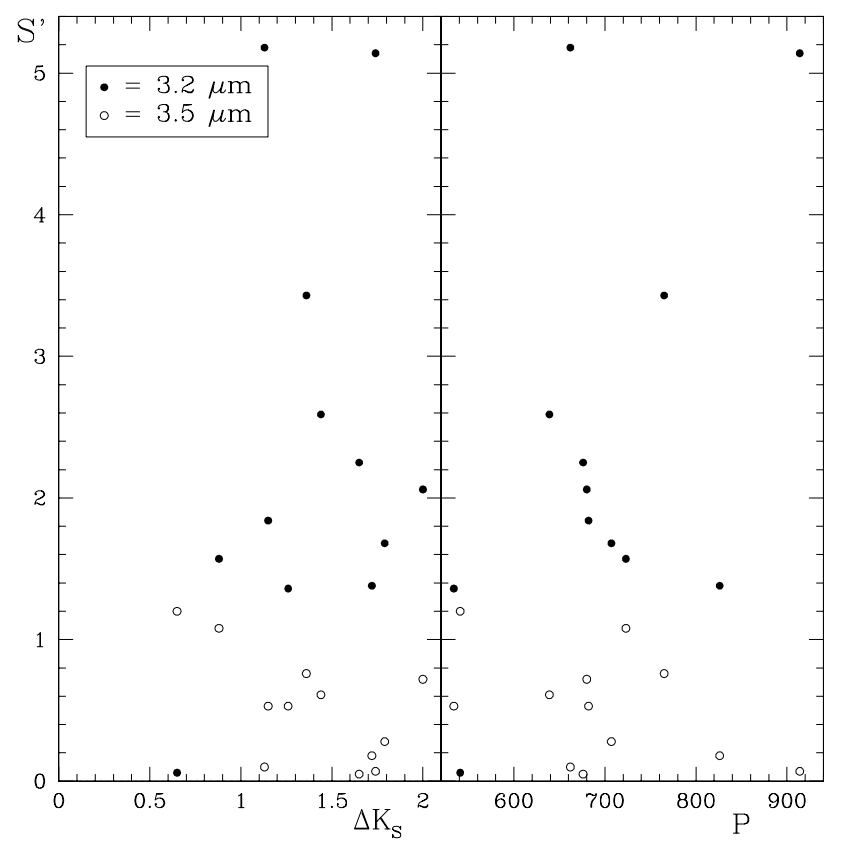

Fig. 20. Change in slope at $3.2 \mu \mathrm{m}$ (solid symbols) and $3.5 \mu \mathrm{m}$ (open symbols) versus $K_{\mathrm{s}}$-band pulsation amplitude (left panel), and versus pulsation period (right panel). The $3.2-3.5 \mu \mathrm{m}$ absorption wing is weaker for larger amplitudes and longer periods, indicating a lower excitation temperature in a more extended molecular atmosphere.

without optical counterparts turns out to almost entirely consist of IR carbon stars (at least 28 out of 30 ).

Allowing for incompleteness in the MSX catalogue due to the detection threshold and confusion in crowded areas or areas with a complex background, the total population of IR carbon stars in the LMC may approach $N_{\mathrm{IR}} \simeq 1000$. This would suggest that, typically, a 1.5-3 $M_{\odot}$ star spends around 10 per cent of its thermal-pulsing AGB lifetime being heavily dust-enshrouded. With mass-loss rates in this superwind stage enhanced by a factor of 10 (or more) over those experienced during the unobscured stages, the mass return during the superwind stage of a carbon star must be (at least) 50 per cent of the total mass returned over its lifetime. This is in excellent agreement with the results found for cluster IR objects (van Loon et al. 2005b).

\subsection{Spectral diagnostics}

The $3.1 \mu \mathrm{m}$ band is due to a mixture of $\mathrm{HCN}$ and $\mathrm{C}_{2} \mathrm{H}_{2}$, but it is difficult to determine the relative contributions of these molecules. In all MSX-selected IR carbon stars in the LMC the $3.8 \mu \mathrm{m}$ band peaks at $3.75 \mu \mathrm{m}$ and is therefore probably due to $\mathrm{C}_{2} \mathrm{H}_{2}$; it never peaks at $3.9 \mu \mathrm{m}$ as it often does in the galaxy due to absorption by HCN and/or CS. The lower metallicity in the LMC would yield lower nitrogen and sulphur abundances, reducing the potential for forming $\mathrm{HCN}$ or $\mathrm{CS}$. The equivalent widths of the 3.1 and $3.8 \mu \mathrm{m}$ bands correlate very well, although the $3.8 \mu \mathrm{m}$ band is not always seen whilst the $3.1 \mu \mathrm{m}$ is always prominent. This suggests that when both bands are strong, the $\mathrm{C}_{2} \mathrm{H}_{2}$ molecule is abundant, whilst $\mathrm{HCN}$ may be more abundant when the $3.8 \mu \mathrm{m}$ band is absent. This is corroborated by the observation that the $3.57 \mu \mathrm{m}$ band due to $\mathrm{HCN}$ is seen both in objects with very strong $3.8 \mu \mathrm{m}$ absorption and in objects without that $\mathrm{C}_{2} \mathrm{H}_{2}$ band. The $3.57 \mu \mathrm{m}$ shows no dependence on luminosity or mass-loss rate, suggesting that its abundance is primarily set by the initially available nitrogen with little evidence for additional nitrogen produced by the $\mathrm{CNO}$ cycle in more massive AGB stars (those would probably not become carbon stars anyway due to Hot Bottom Burning). The strength of the $3.8 \mu \mathrm{m}$ band can thus be taken as a measure for the carbon overabundance resulting from the carbon produced in the star itself, whilst the $3.57 \mu \mathrm{m}$ band measures the (initial) nitrogen abundance.

The $3.1 \mu \mathrm{m}$ band sometimes shows a strong red wing of absorption extending up to $3.5 \mu \mathrm{m}$. Although this could be due to the HNC isomer (Harris et al. 2003), it is not expected to form in great quantities under chemical equilibrium conditions. Unless stellar pulsation causes departures from chemical equilibrium that result in enhanced HNC abundances, it is more likely that the broad absorption is due to a high excitation temperature, closer to the stellar surface. The excitation conditions almost certainly depend on the strength of pulsation, and may vary as a function of phase in the pulsation cycle. We find evidence for the excitation temperature to be on average lower in stars with longer periods and/or larger amplitudes, indicative of a more extended molecular atmosphere.

We have investigated in some detail the nature of the relatively sharp absorptions at 3.70-3.78 $\mu \mathrm{m}$. Of these, the $3.70 \mu \mathrm{m}$ "band" is the easiest to measure reliably. The $3.70 \mu \mathrm{m}$ and associated sharp features are seldomly as prominent in the SWS spectra of galactic carbon stars, suggesting that they may be indicative of the $\mathrm{C}$ enhancement seen in LMC carbon stars. 
The $3.70 \mu \mathrm{m}$ band is generally absent at low mass-loss rates whilst the $3.57 \mu \mathrm{m}$ band is often quite strong in those stars, suggesting that the $3.70 \mu \mathrm{m}$ absorption is not due to $\mathrm{HCN}$. Both $\mathrm{CH}$ and $\mathrm{C}_{2} \mathrm{H}_{2}$ have features in this spectral region. No $\mathrm{CH}$ absorption - either around $3.75 \mu \mathrm{m}$ or elsewhere in the spectrum - is evident in any of the IR carbon stars in the LMC. It is seen around $3.4 \mu \mathrm{m}$ in the optically bright carbon star MSX LMC 349 B (Fig. 4) and in relatively blue carbon stars in Sgr D (Matsuura et al. 2005), but in neither case does absorption at $3.8 \mu \mathrm{m}$ occur. This suggests that in optically bright carbon stars $\mathrm{CH}$ dominates over $\mathrm{C}_{2} \mathrm{H}_{2}$ whilst in IR carbon stars the situation is reversed - probably a consequence of the higher $\mathrm{C}$ overabundance and/or denser atmosphere promoting the formation of larger carbon-chains. The $3.70 \mu \mathrm{m}$ band is strongest at intermediate-strong $3.8 \mu \mathrm{m}$ absorption, and these bands show an opposite behaviour with respect to the $3.2-3.5 \mu \mathrm{m}$ absorption. We suggest therefore that, like the broad $3.8 \mu \mathrm{m}$ band, the 3.70-3.78 $\mu \mathrm{m}$ absorption components are due to $\mathrm{C}_{2} \mathrm{H}_{2}$, with excitation conditions determining their relative strengths in the sense of the sharp features dominating at lower excitation temperature in an extended atmosphere and the broad absorption dominating at higher excitation temperature in lower (or shocked) layers of the atmosphere.

\subsection{The assembly of larger molecules and dust grains}

The properties of the molecular atmosphere amongst the IR carbon stars in the LMC are very homogeneous, and in particular show little - if any - variation with luminosity or massloss rate. For instance, although $\mathrm{C}_{2} \mathrm{H}_{2}$ seems to be abundant amongst LMC carbon stars experiencing the superwind, once in that final evolutionary stage we were unable to identify a strong correlation between the $\mathrm{C}_{2} \mathrm{H}_{2}$ absorption and the massloss rate. The subsequent production of PAH molecules and carbon grains may be largely constant. Veiling of the 3-4 $\mu \mathrm{m}$ spectrum by the continuum emission of warm dust is shown to be important, but it is difficult to accurately account for.

Strong pulsation and intense mass loss do go hand in hand, and we presented some evidence for an extended molecular atmosphere: low excitation temperature, saturated molecular bands, and a possible contribution from absorption seen against the dust envelope. Although this could be thought of as a physical link between the action of pulsation and the dust-driven wind, no clear cause-effect relationship is found. This might indicate that the pulsation and atmospheric structure have already reached the threshold required for complete condensation into grains, leading to a "saturation" of the mass-loss mechanism. That could explain the metallicity-insensitivity of the maximum mass-loss rate attained by AGB stars (van Loon 2000, 2002).

Further insight into the molecule and dust formation should come from a more extensive comparison between oxygen-rich AGB stars and carbon stars in the LMC with those in the SMC, and between the properties of the molecular atmosphere seen at optical and infrared wavelengths with the properties of the dust grains seen at mid-IR wavelengths (Cohen 1984).

\section{Summary of conclusions}

We presented 2.9-4.1 $\mu \mathrm{m}$ spectra, obtained with ISAAC at the ESO/VLT, of a sample of 30 optically invisible stars selected from the MSX-2MASS compilation of Wood \& Cohen (2001). At least 28 (over 90 per cent) turn out to be carbon stars. This confirms earlier indications (van Loon et al. 1997) for an extensive population of IR carbon stars experiencing the superwind stage at the end of the thermal-pulsing AGB. This population is now estimated to account for about 10 per cent of the thermalpulsing AGB lifetime of LMC carbon stars, in agreement with findings from cluster IR objects (van Loon et al. 2005b).

We presented an analysis of the molecular band strengths in the spectra of these and several other LMC carbon stars, and conclude that in the most evolved objects the $\mathrm{C}_{2} \mathrm{H}_{2}$ abundance is large compared to that of $\mathrm{HCN}$ and $\mathrm{CH}$. We interpret this as evidence for a low nitrogen abundance and a high carbon abundance as a result of the low initial metallicity and efficient production of carbon in LMC carbon stars, confirming and extending earlier such evidence (van Loon et al. 1999; Matsuura et al. 2002, 2005).

No strong relationships are found between the strength of the molecular bands and either luminosity or mass-loss rate, but we found hints for the molecular atmosphere to be more extended at the highest mass-loss rates and strongest pulsation, characterised by a low excitation temperature and high $\mathrm{C}_{2} \mathrm{H}_{2}$ abundance. The pulsation, molecular atmosphere and dust wind of these IR carbon stars may have reached their maximum potential for driving mass loss.

Acknowledgements. We would like to thank the Paranal staff for excellent support at the telescope, the anonymous referee for comments which improved the presentation of our results, and Joana Oliveira for help and advice. JRM is supported by a PPARC studentship and MM is supported by a PPARC fellowship. MC thanks NASA for supporting his participation in this work through LTSA grant NAG5-7936 with Berkeley.

\section{References}

Aoki, W., Tsuji, T., \& Ohnaka, K. 1998, A\&A, 340, 222

Cernicharo, J., Yamamura, I., González-Alfonso, E., et al. 1999, ApJ, 526, L41

Cohen, M. 1984, MNRAS, 206, 137

Egan, M. P., Van Dyk, S. D., \& Price, S. D. 2001, AJ, 122, 1844

Gail, H.-P., \& Sedlmayr, E. 1988, A\&A, 206, 153

Harris, G. J., Pavlenko, Ya. V., Jones, H. R. A., \& Tennyson, J. 2003, MNRAS, 344, 1107

Henning, Th., \& Mutschke, H. 1997, A\&A, 327, 743

Ivezić, Ž., Nenkova, M., \& Elitzur, M. 1999, User manual for DUSTY. University of Kentucky Internal Report

Jørgensen, U. G., Larsson, M., Iwamae, A., \& Yu, B. 1996, A\&A, 315, 204

Jura, M., \& Kleinmann, S. G. 1990, ApJ, 364, 663

Kontizas, E., Dapergolas, A., Morgan, D. H., \& Kontizas, M. 2001, A\&A, 369, 932

Latter, W. B. 1991, ApJ, 377, 187

Leisy, P., Dennefeld, M., Alard, C., \& Guibert, J. 1997, A\&AS, 121, 407

Loup, C., Zijlstra, A. A., Waters, L. B. F. M., \& Groenewegen, M. A. T. 1997, A\&AS, 125, 419 
Marshall, J. R., van Loon, J. Th., Matsuura, M., et al. 2004, MNRAS, 355, 1348

Matsuura, M., Zijlstra, A. A., van Loon, J. Th., et al. 2002, ApJ, 580, L133

Matsuura, M., Zijlstra, A. A., van Loon, J. Th., et al. 2005, A\&A, 434, 691

Reid, N., Tinney, C., \& Mould, J. 1990, ApJ, 348, 98

Sanduleak, N., MacConnell, D. J., \& Philip, A. G. D. 1978, PASP, 90, 621

Trams, N. R., van Loon, J. Th., Waters, L. B. F. M., et al. 1999, A\&A, 346, 843

van Loon, J. Th. 2000, A\&A, 354, 125

van Loon, J. Th. 2002, in Radial and Nonradial Pulsations as Probes of Stellar Physics, IAU Coll., 185, ed. C. Aerts, T. R. Bedding, \& J. Christensen-Dalsgaard (San Francisco: ASP), ASP Conf. Ser., 259,548

van Loon, J. Th., Zijlstra, A. A., Whitelock, P. A., et al. 1997, A\&A, 325,585

van Loon, J. Th., Zijlstra, A. A., Whitelock, P. A., et al. 1998, A\&A, 329, 169

van Loon, J. Th., Zijlstra, A. A., \& Groenewegen, M. A. T. 1999a, A\&A, 346, 805

van Loon, J. Th., Groenewegen, M. A. T., de Koter, A., et al. 1999b, A\&A, 351, 559 van Loon, J. Th., Zijlstra, A. A., Bujarrabal, V., \& Nyman, L.-Å. 2001, A\&A, 368, 950

van Loon, J. Th., Marshall, J. R., Matsuura, M., \& Zijlstra, A. A. 2003, MNRAS, 341, 1205

van Loon, J. Th., Cioni, M.-R. L., Zijlstra, A. A., \& Loup, C. 2005a, A\&A, 438, 273

van Loon, J. Th., Marshall, J. R., \& Zijlstra, A. A. 2005b, A\&A, 442, 597

van Loon, J. Th., Oliveira, J. M., Wood, P. R., et al. 2005c, MNRAS Letters, in press

Vandenbussche, B., Beintema, D., de Graauw, T., et al. 2002, A\&A, 390,1033

Whitelock, P. A., Feast, M. W., van Loon, J. Th., \& Zijlstra, A. A. 2003, MNRAS, 342, 86

Wood, P. R. 1998, A\&A, 338, 592

Wood, P. R., \& Cohen, M. 2001, in Post-AGB Objects as a Phase of Stellar Evolution, ed. R. Szczerba, \& S. K. Górny. Ap\&SS Library, 265 (Kluwer Academic Publishers), 71

Wood, P. R., Whiteoak, J. B., Hughes, S. M. G., et al. 1992, ApJ, 397, 552

Yamamura, I., de Jong, T., Justtanont, K., Cami, J., \& Waters, L. B. F. M. 1997, Ap\&SS, 255, 351

Zijlstra, A. A., Loup, C., Waters, L. B. F. M., et al. 1996, MNRAS, 279,32 